\title{
ÉLECTRICITÉ
}

\section{Les Machines électriques à vitesse variable}

Les yrands problèmes de l'industrie moderne: traction, extraction, levage, commande des laminoirs et dès machines dans les différentes industries, ont reçu des solutions diverses avant l'emploi de l'électricité. Leur solution par les machines électriques gagne chaque jour du terrain.

Aussi devient-il nécessaire à l'industriel, quelle que soit l'industrie à laquelle il appartient, de connaîlre les ressources que lui permettent les machines électriques.

Deux questions sont à considérer : les propriẻtés fondamentales des machines, et la spécificalion des machines. Le choix d'une machine ne peut être fait sans cela.

10 Propriétés fondamentales.

L'étude des machines électriques pose différentes catégories de problèmes électrotechniques : problème de bobinage, problème de la rípartition du flux, problème de la commutation. Nous laisserons de côté ces différentes questions pour nous limiter à l'étude des caractéristiques qui définissent les possibilités industrielles de la machine; les autres problèmes n'intéressent que le constructeur.

$2^{\circ} \mathrm{La}$ spécification des machines.

Le service que doit réaliser le moteur étant bien défini par un diagramme, et le genre de moteur élant choisi suivant les indications de la première partie, il reste à définir la machine qui convient pour réaliser ce service. Cette définition, qui fixe les conditions du calcul de la machine, permet aussi de la choisir dans le catalogue du constructeur.

Les considérations qui vont suivre traitent ces deux problèmes au point de vue de l'usager sans théorie algébrique difficile à suivre, mais sans cependant rien sacrifier de la technique, en indiquant toutes les raisons réelles des choses; elles constituent la base nécessaire à l'étude de tous les problèmes d'adaptation du matériel électrique à un service industriel.

PrEMHÈRE PARTIE

\section{LES PROPRIÉTÉS DES MOTEURS}

Chapitre Premier

\section{Les caractéristiques mécaniques des machines}

\section{1. - Les caractéristiques mícanigues DES MOTEURS}

1. - Slabilité el sensibilité d'une machine. - Une machine, moteur ou génèratrice, possède deux caractères importants : la stabilité el la sensibilité (1).

Une machine est stable si, en cas de variation momentanée de l'une des conditions qui fixent son régime de fonctionnement, le régime initial se rétablit de lui-même, antomaliquement, lorsque la perturbation a cessé.

La perturbation entraine l'apparition d'une action correctrice qui lend à rétablir les conditions initiales. Plus cette action correctrice est grande pour une perturbation domée, plus le degré de stabilité est élevé.

Le fonctionnement esl instable si une variation dans un certain sens a pour conséquence un effet qui accentue la variation dans le sens considéré.

Pour étudier les conditions de stabilité, il convient de considérer successivement les différentes variations possibles. Dans la pratique, des petites variations sont toujours possibles : variation accidentelle de vitesse dans le cas d'une machine entrainée

(1) Ces caractères ne sont pas propres à la machine : ils dépendent des conditions de son emploi. à vilesse constante, variation de résistance due à l'échauffement, variation de la charge, ele.

IJ est évident que la stabilité est une condition indispensable au fonctionnement d'une machine.

Une machine est plus ou moins sensible si, en cas de variation persistante de l'une des conditions, l'écart qui en résulte dans les conditions de marche représente un pourcentage plus ou moins important de la valeur initiale.

Si la condition $a$ varie de $\lrcorner a$ et amène une perturbation $\Delta b$ dans la grandeur $b$, la sensibilité est caractérisée par le rapport des variations relatives

$$
\frac{\Delta b}{b} \quad \text { el } \quad \frac{\Delta a}{a}
$$

2. - Point de fonctionnement dun moleur. - Les conditions de stabilité. - Un motcur quelconque à vilesse variable, hydraulique, à vapeur, électrique, possède un couple moteur $C_{m}$ sur son arbre, bien déterminé pour chaque vitesse :

$$
\mathrm{C}_{\mathrm{m}}=f(\mathrm{~N})
$$

cette courbe, obtenue pour une position donnée des organes de réglage, est la caractéristique naturelle du moteur.

Le moteur est utilisé pour surmonter un couple résistant $C_{r}$ qui suit une loi déterminée en fonction de la vitesse

$$
\mathrm{C}_{\mathrm{r}}=(\mathrm{N}) \text {. }
$$

L'intersection des deux courbes donne le point de fonctionnement $\mathrm{P}$.

Les conditions suivant lesquelles les deux courbes se coupent déterminent les conditions de la stabilité du fonctionnement. 
On dira cy'un moteur est stable en cas de variation accidentelle de vitesse si la vitesse reprend automatiquement sa valeur initiale quand les conditions initiales sont rétablies.

Cette condition exige que, cn cas de ralentissement, la différence

$$
\mathrm{C}_{\mathrm{m}}-\mathrm{C}_{\mathrm{r}}=\mathrm{C}_{\mathrm{r}}
$$

soit positive, $\mathrm{C} \gamma$ étant le couple d'accélération. De même, en cas d'accroissement de vitesse, $\mathrm{C}_{\gamma}$ doit ètre négatif.

Il en résulte que si la courbe $q$ est croissante au voisinage du point $\mathrm{P}$ (fig. 1), la courbe $f$ peut avoir l'allure croissante $\mathrm{f}_{1} \mathrm{f}_{1}$

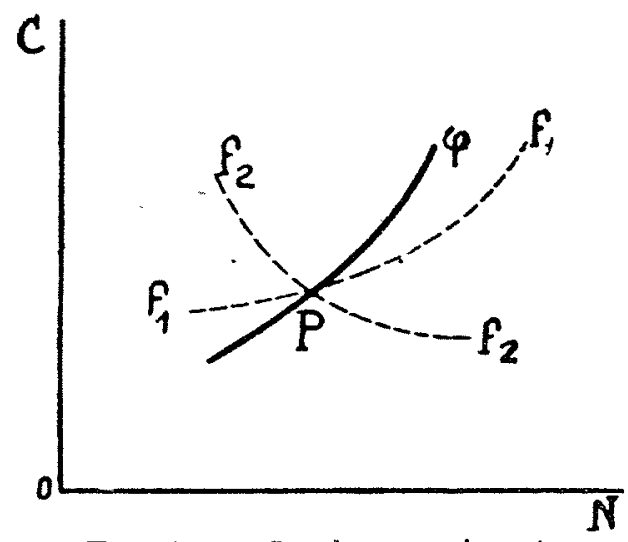

Fig. 1. - Courbe o croissante.

(dans ce cas, le coefficient angulaire de la tangente en $P$ est plus petit pour $f$ que pour $o$, c'est-à-dire que la courbe $\rho_{i}$ est plus -rapidement croissante que la courbe f); ou l'allure $f_{2} f_{2}$ décroissante.

Si la courbe o est décroissante (fig. 2) la courbe ff doit être décroissante avec úne pente plus prononcée.

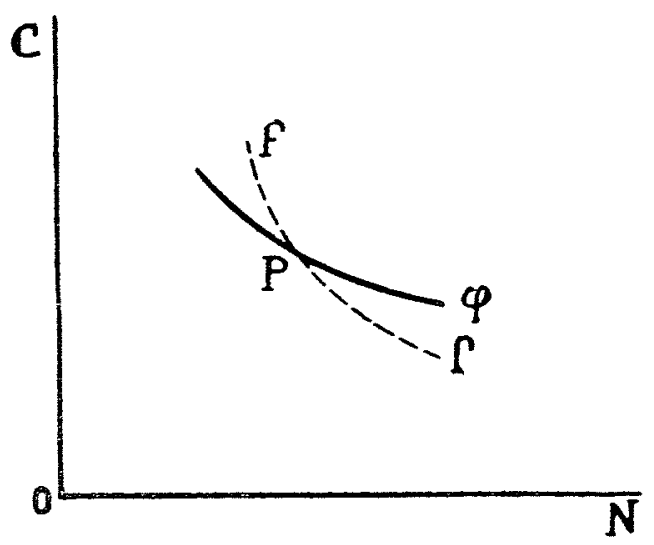

FIG. 2. - Courbe o décroissante.

Enfin, si la courbe $\bullet$ est constante (fig. 3) la courbe $f f$ doit être décroissante.

Le degré de stabilité du moteur, qui caractérise la rapidité du retour aux conditions initiales, après une perturbation, est mesuré par la grandeur de l'angle des courbes $f$ et o au point $P$. $\mathrm{Si}$ cet angle ést nul, le couple correcteur $\mathrm{C}_{\gamma}$ est très faible pour une petite variation accidentelle de la vitesse; les conditions initiales ne se rétablissent que très lentement (ou même pas du - tout si les deux courbes sont confondues : la vitesse est alors indéterminée).

Si le moteur est accouplé à sa charge à l'arrêt, le démarrage n'a lieu que si l'ordonnée à l'origine de $f$ dépasse celle de $o$; le moteur accélère alors jusqu'au point $P$.

Inversement, aux grandes vitesses, le moteur ayant atteint sa vitesse à vide à la suite de l'annulation du couple résistant, si l'on rétablit brusquement la charge, il ne peut y avoir ralentissement que si la courbe de $q$ est au-dessus de celle de $f$.

Dans la pratique, il est rare d'avoir plusieurs points d'intersection entre les courbes $f$ et $\varphi$; mais, en pareil cas, on recon-

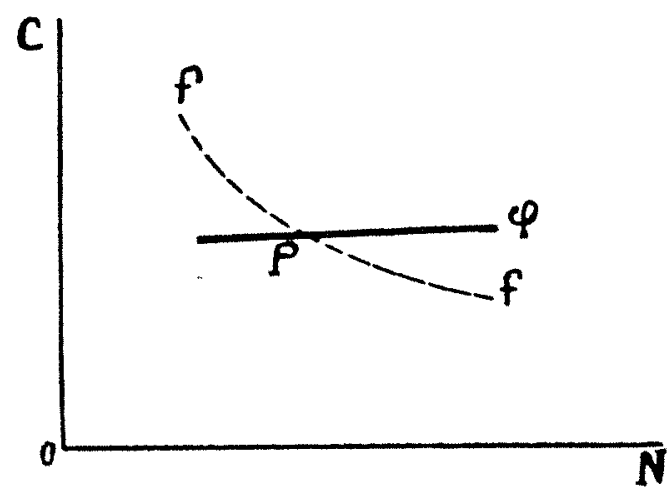

FIG. 3. - Courbe o constante.

naît facilement comme ci-dessus les points stables et les points instables.

Si le couple moteur passe par un maximum pour une certaine vitesse, et que le couple résistant vienne à dépasser ce maximum, il n'y a plus de point de fonctionnement possible : le moteur décroche.

3. - Moteuf à vitesse réglable. - On appelle moteur à vitesse réglable, un moteur capable de différentes caractéristiques naturelles, obtenues en modifiant la position d'un organe de réglage (admission dans le cas d'une lurbine, rhéostat ou décalage des balais dans le cas d'un moteur électrique). Sur chacune de ces caractéristiques, la charge normale, c'est-à-dire la charge pour laquelle les circuits électriques et magnétiques sont le mieux utilisés, correspond à un point bien déterminé $\mathrm{P}$; le lieu des points de pleine charge P sur les différentes caractéristiques, est une courbe $\psi$ que nous appellerons caractéristique d'utilisation normale du moteur à vitesse réglable (fig. 4).

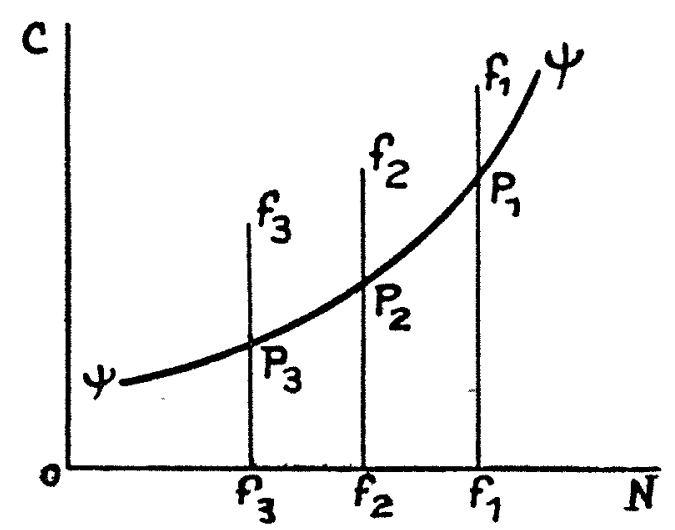

FIG. 1. - Caractéristique d'utilisation normale.

Ce sera, par exemple : une droite, si le moleur est capable du même couple à toutes les vitesses; un arc d'hyperbole, s'il est capable de la même puissance à toutes les vitesses; et, parfois, une courbe plus compliquée.

4. - Fonctionnement à charge variable. - Nous avons considéré jusqu'ici le cas du fonctionnement à pleine charge. Mais un moteur est souvent appelé à fonctionner à charge variable, selon le service à réaliser.

Si la charge peut varier de façon continue à partir de la marche à vide, on pourra obtenir en marchë de régime, chacun des points de la caractéristique $f$ correspondant au réglage actuel du moteur. 
Pour un réglage donné, le moteur à charge variable suivra sa caractéristique naturelle.

5. - Chute de vitesse. - La chute de vitesse d'un moteur utilisé à charge variable (ou écart entre la vitesse à vide et la vitesse à pleine charge pour un réglage déterminé) caractérise l'allure d'une caractéristique naturelle.

Si la caractéristique permet de réaliser une vitesse pratiquement constante quand la charge varie, on dira que la caractéristique est shunt (fig. 5); si elle donne au contraire une vitesse

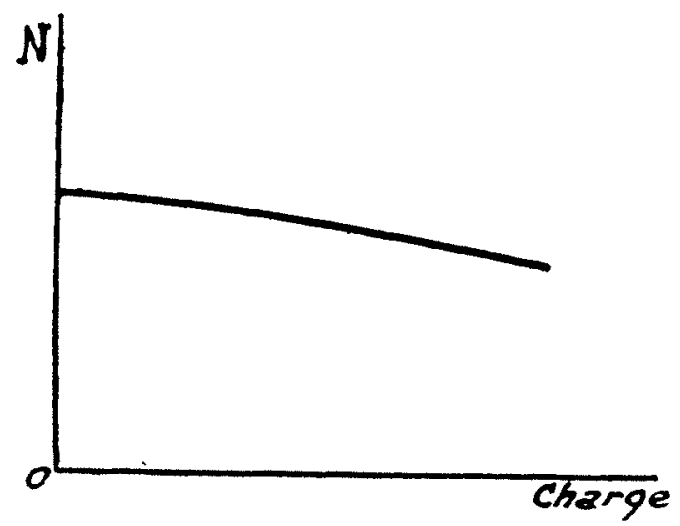

FIG. 5. - Caractéristique shunt.

qui baisse franchement quand la charge augmente, on dit que la caractéristique est série (fig. 6) ou compound (fig. 7); (le

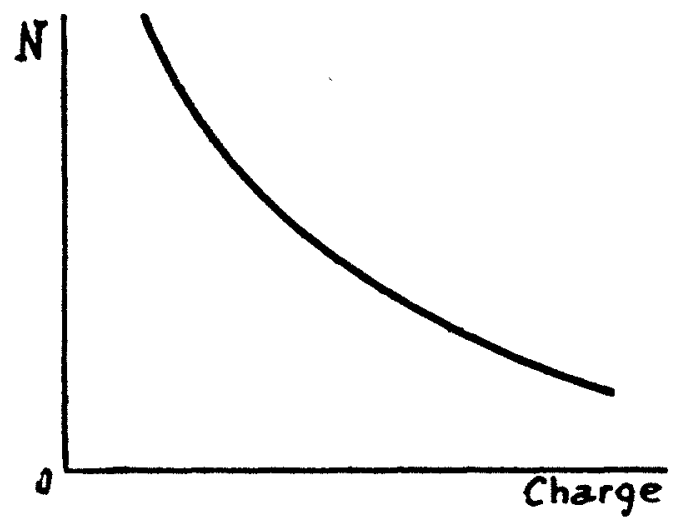

FIG. 6. - Caractéristique série.

terme compound s'appliquant plus spécialement au cas où la vitesse est limitée à vide).

Nous reviendrons par la suite sur la raison pour laquelle les caractéristiques sont ainsi nommées.

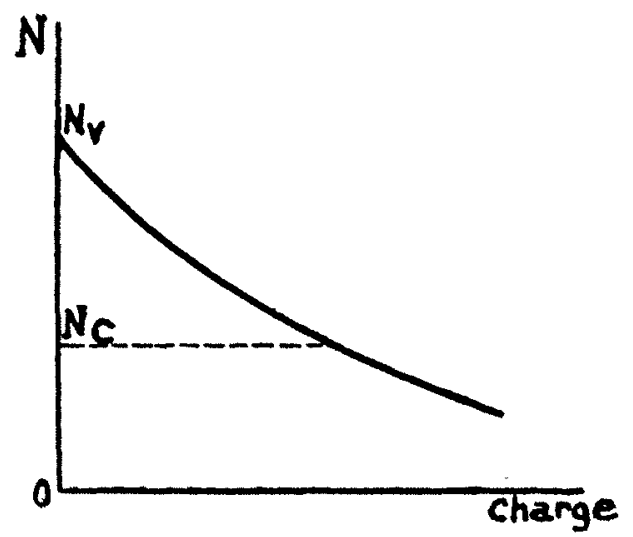

FIG. 7. - Caractéristique compound.

Selon la nature de la charge accouplée au moteur, ce sera l'une ou l'autre caractéristique qui conviendra; la vitesse devant; de toute façon, être limitée à vide si la charge peut s'annuler.

La caractéristique série ne convient pas si l'on désire obtenir la même vitesse quelle que soit la charge, pour une position donnée de l'organe de réglage.

Elle est parfois recherchée pour des raisons d'auto-régulation : quand la charge augmente pour une raison accidentelle, la vitesse baisse, le couple moteur augmente et tend à vaincre la surcharge pour rétablir l'état initial.

Entre la caractéristique shunt et la caractéristique compound, la différence n'est pas nettement définie; la grandeur à considérer est le rapport

$$
\frac{\Delta \mathrm{N}}{\Delta \mathrm{C}}
$$

de la variation de vitesse à la variation de charge qui cause cette variation de vitesse.

Ce rapport varie avec la région de la caractéristique que l'on considère si celle-ci n'est pas rectiligne.

6. - Caractéristique puissance-vitesse. - Considérons la caractéristique $(C)$ de la vitesse en fonction de la puissance du moteur.

On a la relation :

$$
\mathrm{P}=\mathrm{C} \omega=\mathrm{C} \frac{2 \pi \mathrm{N}}{60}
$$

Considérons une droite AE parallèle à l'axe horizontal (fig. 8)

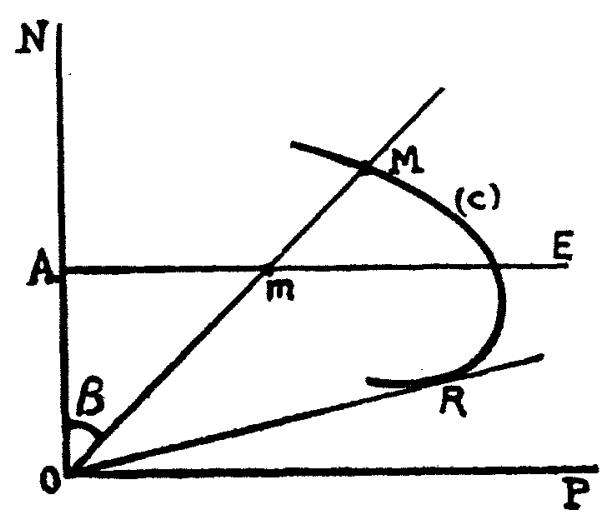

FIG. 8 . - Caractéristique puissance-vitesse.

et soit $m$ l'intersection de cette droite avec le rayon vecteur abaissé du point $M$ de la caractéristique sur l'origine $O$.

On a :

$$
\operatorname{tg} \beta=\frac{\mathrm{A} m}{\mathrm{OA}}=\frac{\mathrm{P}}{\mathrm{N}}=\frac{2 \pi}{6 \mathrm{U}} \mathrm{C} .
$$

Le couple est donc mesuré par le segment Am. La droite AE peut être gradué en couples à partir de l'origine $\mathrm{A}$, et permet de trouver immédiatement le couple en tout point de la caractéristique. Ainsi, le couple est maximum au point $R$ où le rayon vecteur $O M$ est tangent à $(\mathrm{C})$.

On voit que les caractéristiques couple-vitesse et puissancevitesse n'ont pas la même allure; le couple étant donné par la courbe

$$
\begin{gathered}
\qquad y_{1}=f(\mathrm{~N}) \\
\text { la puissance sera de la forme } \\
y_{2}=\mathrm{N} f(\mathrm{~N}) .
\end{gathered}
$$

La courbe $y_{1}$ ne passe pas, en général, par l'origine tandis que la courbe $y_{2}$ y passe ; les courbes $y_{1}$ et $y_{2}$ ne passent pas par leur maximum pour la même valeur de $N$.

En particulier, si la caractéristique couple-vitesse est une 
droite, on démontre alsément que la caractéristique pulssanco vitesse est une parabole :

Soit $\mathrm{C}_{\mathrm{d}}$ le couple au démarrage, et $\mathrm{N}_{\mathrm{v}}$ la vitesse à vide. L'équation de la caractéristique couple-vitesse est (fig. 9)

$$
\frac{N}{N_{\mathrm{v}}}+\frac{C}{C_{d}}=1
$$

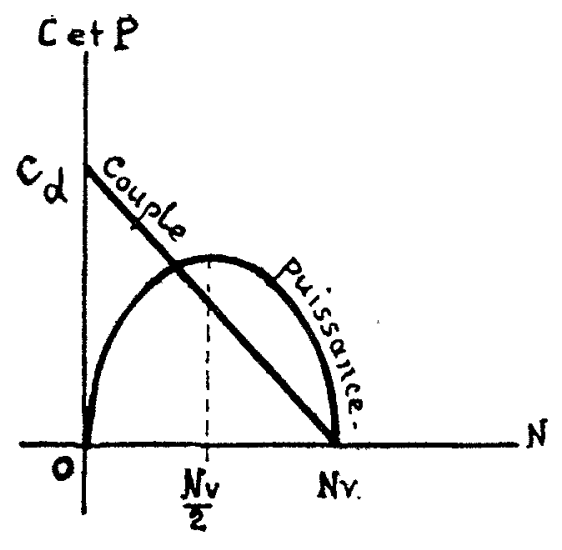

FIG. 9.

L'équation de la caractéristique puissance-vitesse est :

$$
\mathrm{P}=\mathrm{C} \times \frac{2 \pi \mathrm{N}}{60}
$$

En éliminant $\mathrm{C}$ entre ces deux relations, on voit que la courbe de $\mathrm{P}$ en fonction de $\mathrm{N}$ est une parabole dont l'ordonnée s'annule pour $\mathrm{N}=\mathrm{O}$ et $\mathrm{N}=\mathrm{N}_{\mathrm{v}}$ et passe par un maximum pour

$$
\mathrm{N}=\frac{\mathrm{N}_{\mathrm{v}}}{2}
$$

Ainsi, dans le cas où le moteur que l'on considère est une turbine, la caractéristique couple-vitesse dépend d'un paramètre qui est le degré d'ouverture du vannage.

La caractéristique est sensiblement rectiligne, et se déplace vers l'origine des coordonnées quand l'ouverture diminue (fig. 10).

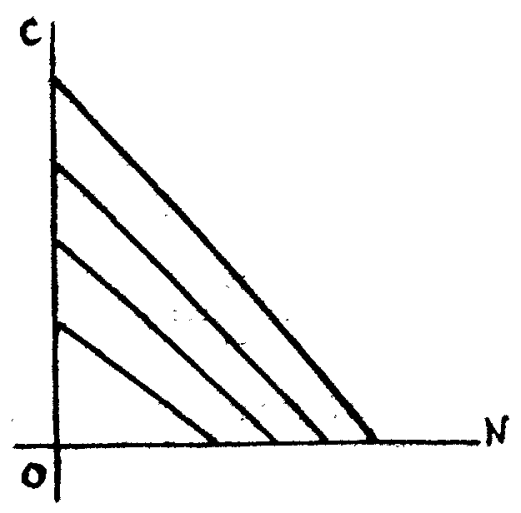

FIG. 10.

La caractèristique puissance-vitesse s'en déduit, suivant l'allure parabolique (fig. 11).

Le rendement de la turbine est, en général, maximum en même temps que la puissance : on voit ainsi que la vitesse optimum de fonctionmement d'une turbine est voisine de sa demivitesse d'emballement.

Selon que la caractéristique couple-vitesse est d'allure shunt ou d'allure série, la puissance suit une loi différente en fonction da couple.

Avec la caractéristique shunt, la puissance utile est sensiblement proportionnelle au couple; avec la caractéristique série, dile vario peu avec le couple (elle est indépondante du couple dans le cas de la caractéristique série hyperbolique : couple inversement proportionnel à lia vilesse).

Or, d'une part, il y a intérêt, en général, à ce que la puissance absorbée par un moteur soit aussi conslanle que possible lorsque la charge varie. D'autre part, une caracléristique trop série conduirait à un ralentissement inadmissible pour la production.

Il faut, dans la pratique, concilier au mieux ces différentes conditions.

7. - Passage de la marche en moleur à la marche en générulrice. Il arrive souvent, pour une machine qui possède une vilesee à vide déterminée, que le fonctionnement soil réversible : pour toute vitesse inférieure à la vitesse à vide, le fonctionnement est en moteur; aux vitesses supérieures, il est cn génératrice. La caractéristique couple-vilesse est continue en passanl des couples positifs (moteur) aux couples négatifs (génératrice).

8. - Caractéristique d'un moteur avec régulateur de vilesse. $\mathrm{Au}$ lieu d'utiliser la caractéristique naturelle, on est conduil parfois à utiliser un régulateur, automatiequé contrôlé par la vitesse.

Le rôle du régulateur de vilesse d'un moleur esl de mainte-

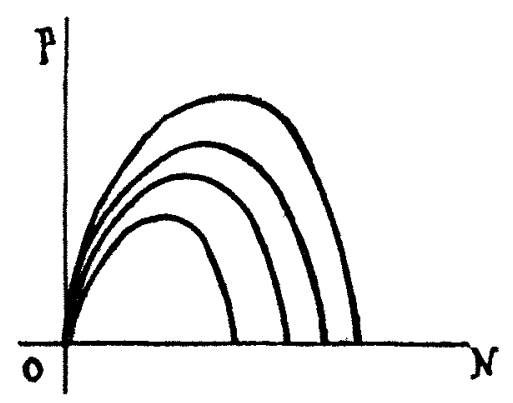

FIG. 11.

nir la vitesse du moteur constante lorsque la charge varie : on dit alors que le régulateur est isochrone.

On demande parfois áu régulateur de vitesse d'imposer une relation déterminée entre la vilesse d'une machine et sa charge: cette relation se traduit par une courbe que l'on appelle caractéristique de statisme.

Le plus souvent on impose la condition que la vitesse diminue quand la charge augmente : la caractéristique de statisme est une droite inclinée d'allure shunt.

On dira que celte caractéristique est réglable, si on peul, soil en changer l'inclinaison, soit la déplacer parallèlement à ellcmème.

Un régulateur est caractérisé en outre par son degré d'insensibilité : il faut une certaine variation de la vitesse pour que le régulateur commence à agir. La caractéristique de statisme, n'est pas une ligne sans épaisseur : c'est une bande étroite donl la largeur caractérise l'insensibilité.

\section{H. - LES CONDITIONS PARTICULIÈRES AUX MACHINES ÉLECTRIQUES}

Les conditions indiquées dans le paragraphe précédent, s'appliquent à toutes les catégories de moteurs. Nous allons considérer maintenant les caractéristiques particulières aux machines électriques.

1. - Caractéristique couple courant. - Dans un assez grand nombre de catégories de moteurs électriques, le couple moteur 


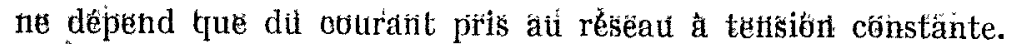

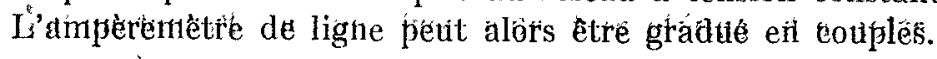

Le códiple à cobinsidérer est lé côuplé mécảniqué $\mathrm{C}_{\mathrm{m}}$ sur l'arbìte

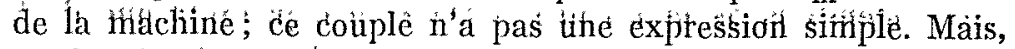

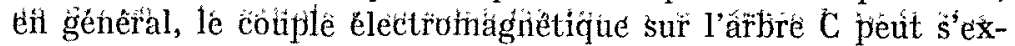
primer d'une faegort simple en fonction des courants et des flux de la machine; el it existe une relation entre $\mathrm{C}$ et $\mathrm{C}_{\mathrm{m}}$.

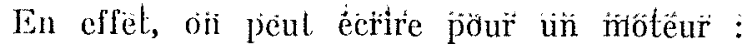

$$
\mathrm{C}_{\mathrm{m}}=\dot{\mathrm{C}}-\mathrm{C}_{\mathrm{p}}
$$

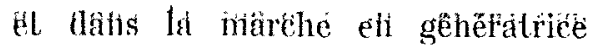

$$
\mathrm{C}_{\mathrm{m}}=\mathrm{C}+\mathrm{C}_{\mathrm{p}}
$$

$\mathrm{Cp}_{p}$ étant le couple correspondant aux pertes mécaniques (frottements et ventilation) el aux pertes dans le fer. Si l'on désigne par $p$ la somme de ces pertes et $\omega$ la vitesse angulaire.

$$
\mathrm{C}_{\mathrm{p}}=\frac{p}{\omega}
$$

C pourra être pris comme expression approchée de $\mathrm{C}_{m}$; l'allure des variations ne changera pas.

2. - Dtagramme de courant: - Coñsldêrons utiè nòachine

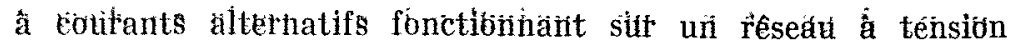
constante.

Cette machine prend au réseau une puissance électrique $P_{w}$ et échange avec lui une puissance déwattée $\mathrm{P}_{\mathrm{dw}}$.

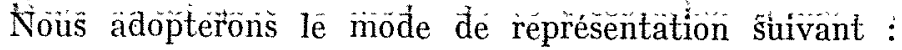

Prenons deux axes OX OYY, l'axe ỡ étant l'axe des puissances waltées et OY l'axe des puissances déwattées. Ces axes

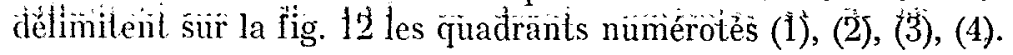

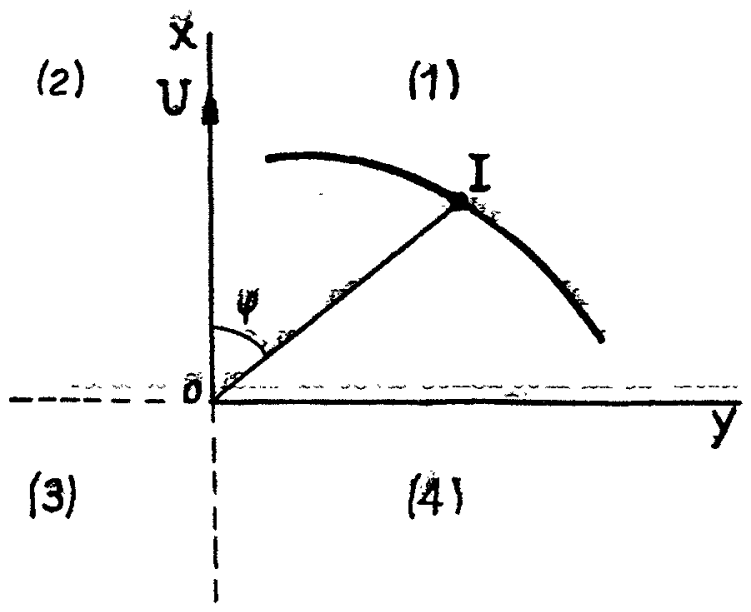

FIG. 12. - Diagramme de courant.

Comme nous considérons le fonctionnement sur réseau à tension constante, on peut parlei de courant watté ou déwatté aussi bien que de puissance wattée ou déwattée, ces deux grandeurs étant proportionnelles : les échelles seules diffèrent. (En triphasé $P=U$ I $V$ 3:)

La tension du réseau sera portée suivant $O X$ : Le courant I pris au réseau sera décalé d'un angle $९$ par rapport à U. Si I

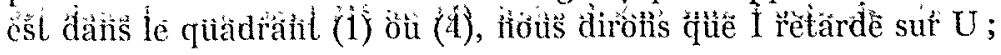
le décalage est en arrière.

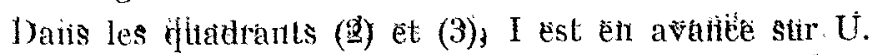

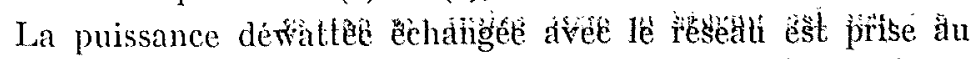
réseau dans les quadrants (1) et (4); elle est fournie au réseau dans les quadrants (2) ei (3).

In puissance wattee échangée avec le réseau cst prise aut résenu

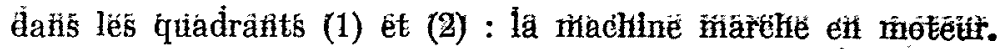

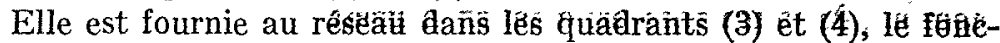
tionnèment est générateur.

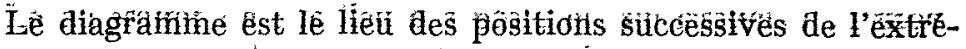

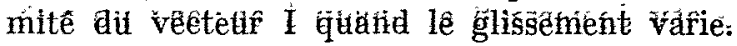

Sur le diagramme, on lit donc immédiatement êt tơtut prifit de fơnctionnement le eoúrant; la pulssance wattees la puissance déwattée, le déphasage, et on reconnaît le mode de fontetionnement (moteur ou générateur).

İl reste à représentēr le coup̃le et la vítesse.

Couple. - Dans chaque cas particulier, ón peut définir une

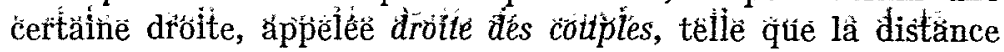
d'un peint du diagramme à la droite des couples, comptée suivant une direction fixe, mesure le couple au point considéré du diagramme.

Vitesse. - A ehaque point di diagramme córrespond unie certaine vitesse : au démarrage, le point de fonctionnement est en un point tel que $D$ (fig. 13), et à mesure que la vitesse

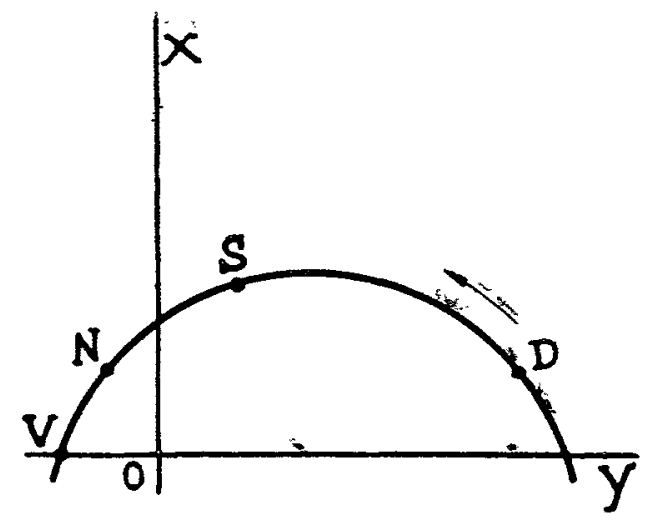

FIg. 13. - Points du diagramme cơrrespontiant aux différentes vitesses.

augmente, le pôtit de fonctionnement avante sur le diagramme dans le sens de la flèche. Au synchronisme correspond le point S; à la charge normale le point $\mathrm{N}$; à la marche á vide le point $\mathrm{V}$ intersection du diagramme avec l'axe OY.

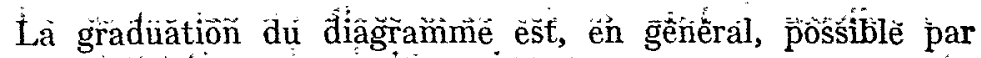

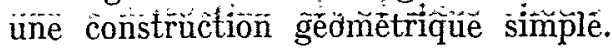

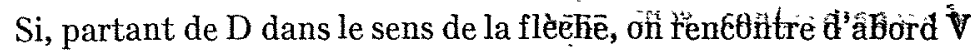
puis $\mathrm{S}$, cela veut dire que la vitesse à vide est hyposynchrone.

En conclusion, le diagrammë, complété par les points $\breve{D}, \breve{N}, S$,

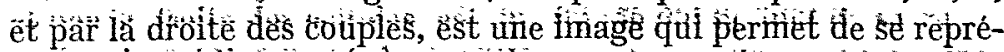

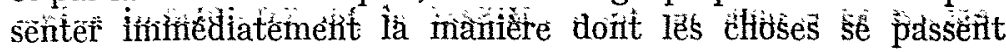
pendant le fonetionnement:

L'allure de la courbe, et la position des points caractéristiques, varient peu avec les dimensions particulieres, poưr un genre

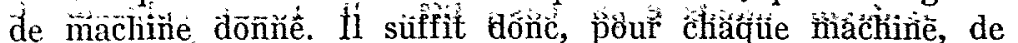

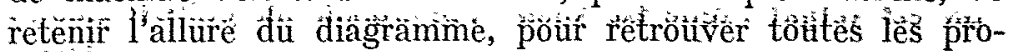
priétèts.

3. - Variation de la tension et de la fréqueñce. - Dans le cas d'un moteur alimenté par un réseau à tension et fréquence constantes, il importe de considerer l'effet d"une variation accidentelile de la tension, ou de la fréquêtice, sur lès caractêrístiques.

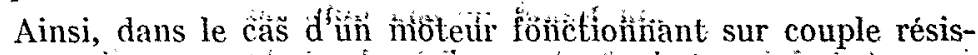

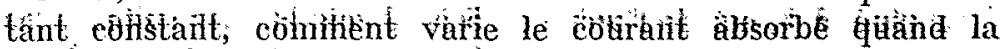

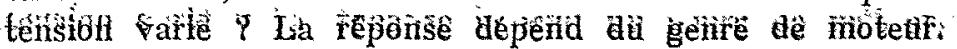

$$
\text { 4. Démấrage. - Nous désignerons pãr c le rapport du }
$$


couple de démarrage au couple normal, et par $i$ le rapport du courant de démarrage au courant normal.

Si on désire surmonter au démarrage le couple résistant normal, il faudra que le moteur développe un couple supérieur au couple normal, afin de permettre l'accélération ; on aura $c=1,2$ par exemple.

La courbe couple-courant permettra de déterminer le rapport $i$.

On considère souvent le rapport $\frac{c}{i}$, qui caractérise les conditions au démarrage.

$\mathrm{Si}$, par exemple, la courbe couple-courant est une droite, on aura $\frac{c}{i}=1$. Si la courbe est une parabole du second degré (cas du moteur série à courant continu non saturé), on aura $\frac{c}{i}=i$. En prenant 2 fois le courant normal, on aura 4 fois le couple normal.

5. - Puissance déwattée. - En courants alternatifs, la puissance déwattée joue un rôle important, comme il résulte de la comparaison suivante :

Comparons deux moteurs produisant la même puissance utile, mais le moteur 1 ne consommant aucune puissance déwattée et le moteur 2 consommant la puissance déwattée $\mathrm{AB}$ (fig. 14).

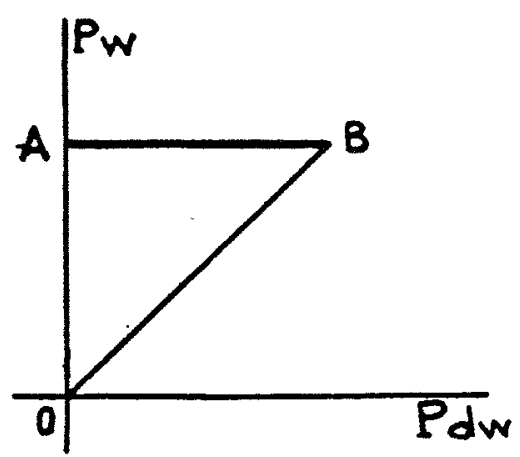

FIg. 14. - Comparaison des courants absorbés.

Le moteur 1 prend au réseau le courant total OA et le moteur 2 le courant total $O B$. Son facteur de puissance est le cosinus de l'angle $\mathrm{AOB}$, soit $\frac{\mathrm{OA}}{\mathrm{OB}}$.

On peut alors faire les remarques suivantes :

$1^{\circ}$ Le circuit parcouru par le courant qui alimente le moteur - est traversé dans le cas du moteur 2 par un courant majoré dans le rapport $\frac{\mathrm{OB}}{\mathrm{OA}}$. Les pertes Joule sont majorées suivant le carré de ce rapport.

Le circuit intéressé par ces pertes comprend le primaire du moteur (organe relié au réseau); le transformateur éventuel, la * ligne de distribution; l'induit de l'alternateur à la centrale.

Quand on emploie le moteur (2) au lieu du moteur (1), les pertes totales sont donc sensiblement majorées.

$2^{\circ}$ Quand un courant passe dans un circuit, le passage du courant est accompagné d'une chute de tension; si $\mathrm{R}$ est la résistance et $l_{\omega}$ la réactance, la chute totale est

$$
\mathrm{RI} \text { watté }+l_{\omega} \mathrm{I} \text { déwatté }
$$

en général $l_{\omega}$ est de l'ordre de 5 à 10 fois R. Par suite, le passage d'un courant ayant une composante déwattée appréciable détermine une grande chute de tension en passant dans la ligne et dans le transformateur.
$3^{0}$ Un alternateur qui fournit seulement de la puissance wattée est sensiblement plus petit.qu'un alternateur aúquel on demande, en outre, de la puissance déwattée. Tandis que les dimensions de la turbine motrice ne dépendent que de la puissance wattée, celles du primaire de l'alternateur dépendent de la puissance apparente et sont par suite $\frac{\mathrm{OB}}{\mathrm{OA}}$ fois plus grandes; et celles du rotor augmentent plus rapidement encore.

En conclusion, le moteur 2 est plus conteux à alimenter pour le producteur d'électricité que le moteur 1 ; il est logique que l'on fasse payer au consommateur la puissance déwattée consommée.

6. - Marche en parallèle. - La marche en parallèle de deux machines sur une charge commune, pose trois problèmes fondamentaux :

10 Le problème de la répartition entre les deux machines d'une charge totale donnée.

$2^{\circ}$ Le problème de la stabilité de ce partage.

$3^{\circ}$ Le problème de la modification de la répartition des charges quand la charge totale varie.

Remarquons d'abord que, quand deux machines fonctionnent en parallèle, elles sont assujetties à une condition d'égalité particulière.

Considérons, pour fixer les idées, trois exemples simples :

a) Deux génératrices à courant continu couplées en parallèle sur un réseau sont assujetties à la condition d'égalité de tension aux bornes.

L'étude du problème résulte du tracé des caractéristiques tension-courant, qui permet d'étudier le partage des charges et d'établir la loi de la tension aux bornes en fonction de la charge totale.

b) Deux turbines entraînent 2 génératrices synchrones couplées en parallèle.

Le couplage électrique imposé la condition d'égalité des vitesses.

L'étude du problème résulte du tracé des caractéristiques de statisme, qui permet d'étudier le partage des charges et la loi de variation de la fréquence avec la charge totale.

c) Deux moteurs électriques rigidement accouplés entraînent une même charge. L'accouplement impose une vitesse commune.

L'étude du problème résulte du tracé des caractéristiques mécaniques.

Pour résoudre les problèmes posés, il suffit de se donner les caractéristiques $y=f(x)$ de chaque machine, $x$ représentant la charge (courant dans le premier exemple, puissance dans les deux autres), et $y$ la grandeur qui en dépend (tension dans le premier exemple, vitesse dans les deux autres). Souvent, ces caractéristiques sont réglables (réglage de l'excitation, dans le premier cas; réglage du régulateur, dans le second).

On peut alors énoncer les propositions suivantes :

10 La répartition des charges est déterminée par l'intersection des caractéristiques.

$2^{\circ}$ La condition pour que la marche en parallèle soit stable, est que les caractéristiques soient tombantes.

$3^{\circ}$ Une machine est d'autant plus sensible, c'est-à-dire d'autant plus influencée par les variations de la charge totale, que sa caractéristique est moins tombante. 
Considérons, en effet, les caractéristiques respectives (fig. 15)

$$
y=f(x) \quad \text { et } \quad y^{\prime}=f\left(x^{\prime}\right)
$$

et $\mathrm{X}=x+x^{\prime}$ la charge totale.

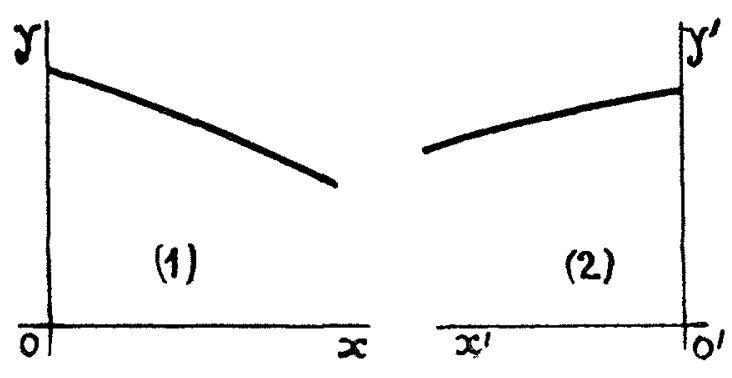

FIG. 15.

Supposons la charge totale donnée à un instant déterminé, soit $X_{0}$ sa valeur : .

$$
\mathrm{X}_{\mathrm{o}}=x_{\mathrm{o}}+x_{\mathrm{o}}^{\prime}
$$

Rapportons la courbe $y=f(x)$ aux axes $y o x$, et la courbe $y^{\prime}=f\left(x^{\prime}\right)$ aux axes $y^{\prime} o^{\prime} x^{\prime}$, de telle façon que les axes des $y$ soient parallèles et de même sens, et les axes de $x$ confondus et de sens opposés.

Le point $O$ étant fixe, nous ferons glisser $\mathrm{O}^{\prime}$ de manière que la distance $O O^{\prime}$ représente la charge totale (fig. 16).

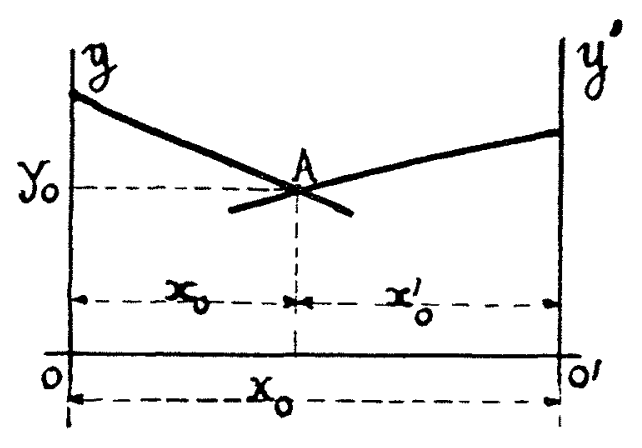

FIG. 16.

Le point d'intersection $\mathrm{A}$ des deux courbes détermine les charges respectives $x_{0}$ et $x_{0}^{\prime}$ des deux machines et l'ordonnée commune $y_{0}$ du point de fonctionnement.

Etudions alors la stabilité.

$\mathrm{X}_{0}$ étant donné, supposons que, pour une raison quelconque, la machine (1) vienne à débiter une charge plus grande $x_{1}$.

La machine (2) doit alors débiter le complément $x_{1}^{\prime}$. Cet état de choses ne peut persister, les points de fonctionnement étant assujettis à se trouver sur les caractéristiques respectives et à leur intersection, pour tout régime stable.

De deux choses l'une : ou bien l'état initial se rétablit automatiquement (régime stable), ou bien le déréglage s'accentue.

On se trouve, dans le premier cas, quand les caractéristiques ont l'allure tombante, et dans le second quand elles ont l'allure montante.

Pour le démontrer nous admettrons que la machine pour laquelle y est le plus grand tend à se charger aux dépens de l'autre machine qui se décharge.

Il est dès lors évident que, quand les caractéristiques sont tombantes (fig. 17), la machine 1 tend à se décharger, ce qui rétablit l'état initial; quand elles sont montantes, la machine 1 tend à se charger davantage, ce qui a pour conséquence un nouvel accroissement de la charge, etc. (fig. 18).

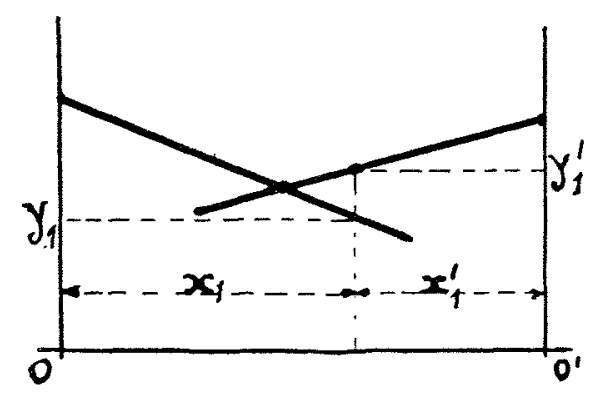

FIG. 17.

Etudions maintenant la variation de la répartition des charges lorsque la charge totale varie.

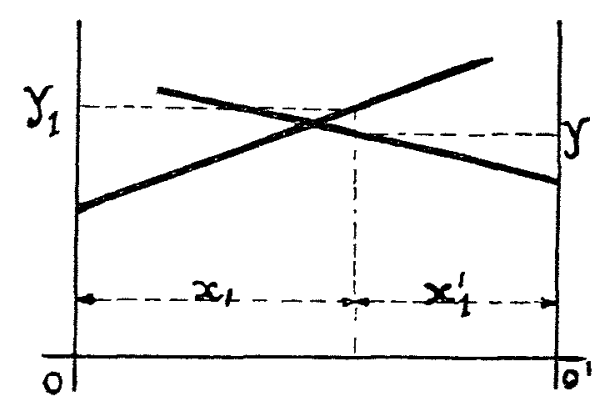

FIG. 18.

Cette répartition dépend de l'inclinaison relative des caractéristiques.

Si la charge $\mathrm{X}$ passe de $\mathrm{X}_{1}=x_{1}+x_{1}^{\prime}$ à $\mathrm{X}_{2}=x_{2}+x_{2}^{\prime}$, on voit que la machine dont la caractéristique est la moins inclinée supporte presque toute la variation de la charge (fig. 19).

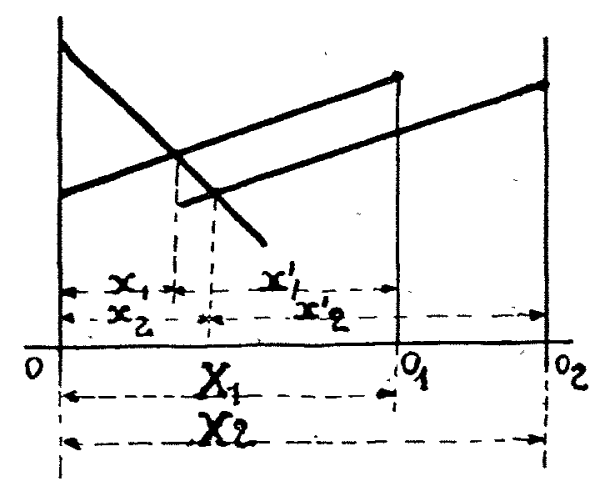

FIG. 19.

En outre, il faut remarquer que, lorsque les deux caractéristiques sont très peu inclinées, une petite modification accidentelle de l'une des caractéristiques a pour effet une grande variation dans la répartition des charges : le fonctionnement est alors très sensible.

Lorsque l'inclinaison del'une des caractéristiques est réglable, il est possible de modifier l'influence sur cette machine des variations de la charge totale.

Lorsque la caractéristique peut être déplacée parallèlement à elle-même, il est possible de modifier la répartition des charges pour une charge totale arbitrairement choisie; pour toutes les autres charges, le partage des charges est alors déterminé. 
En particulier, il est possible de faire passer progiressiveinent la charge de la machine 1 suir la machine 2 en dépläçant sa cúfactéristique de $a a^{\prime}$ en $b b^{\prime}, c c^{\prime}$, etc... (fig. 20).

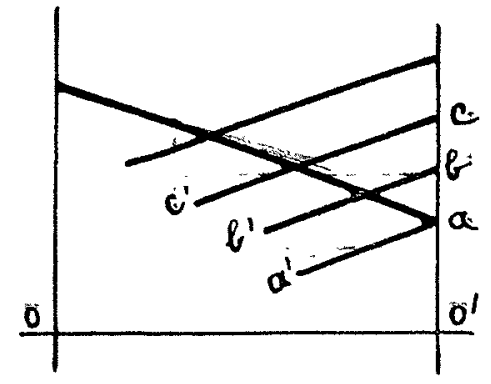

Fig. 20.

\section{Chapitre II}

\section{Les moteurs à courant continu}

1. - Allure des caractéristiques. - Les moteurs à courant continu alimentés par̉ un réseau à tension constante, se distinguent par l'allure de leur caractéristique en fonction de la vitesse.

A) Moteur sérié, le couple varie en sèns inverse de la vitesse, le moteur s'emballant à vide (fig. 6).

B) Moteur shunt, le couple est pratiquement indépendant de la vitesse; à vide; le moteur prend une vitesse $\mathrm{N} v$ qui diminue légèrement quiand la charge augmente (fig. 5).

Ces allures de caractéristiques sont bien différenciées, et on en est venu, comme nous l'avons indiqué au chapitre I, à donner le nom de moteur à caractéristique série, à caractéris-

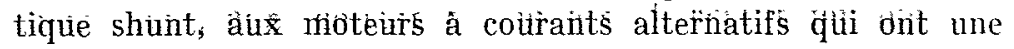
courbe couple-vitesse de même allure que celle du hiöteili: à courant continu de même nom:

On appellera encore moteur à caractéristiquie série un móteur à vitesse limitée à vide tel que le moteur compound courant continu à enroulement série prépondérant.

c) Nous appellerons plus spécialement moteur à caractéristique compound̆, un moteur à vitesse limitée

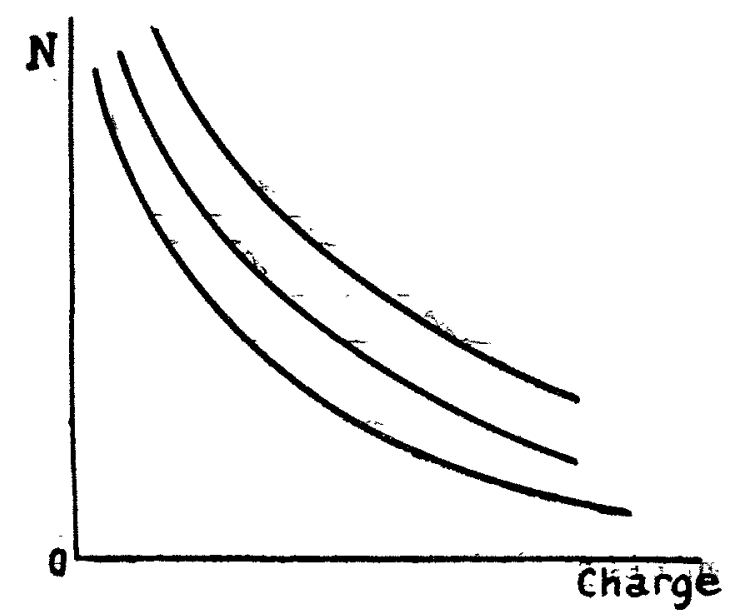

FIG. 21: - Moteur série cc: (réglagé par shuntage): Caractéristicques série réglable.

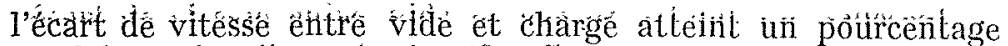
setisisiblè dé la vitessé

Cette caractéristique est donc intermédiaire entre les caractếristiqứes sériè̉ êt sliurit. Elle peut êtré réáliŝêe èn particulier

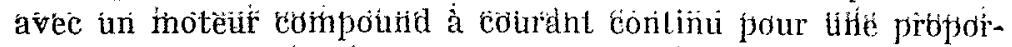

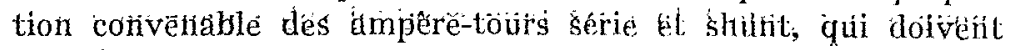
êtré conticơrdantits:

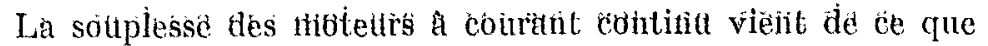
leur caractéristique couple-vilesse peut être modifiée par différents moyens : de sont des móleurs à ćardctéristique réglable.

Ce réglage peut se faire par le champ, ou par résistance en séric, dans les cas les plus simples. Selon le moyen employé, la caractéristique est différente. L'ensemble des caractéristiques obtenues conistitue un faiscenu de courbes qui définit les possibilites du motetir.

Ainsi, le moleur sètie avec réglage par shuntage ou par résistance en sceric aura un faisceau de courbes d'allute (fig. 21 ou lig. 22).

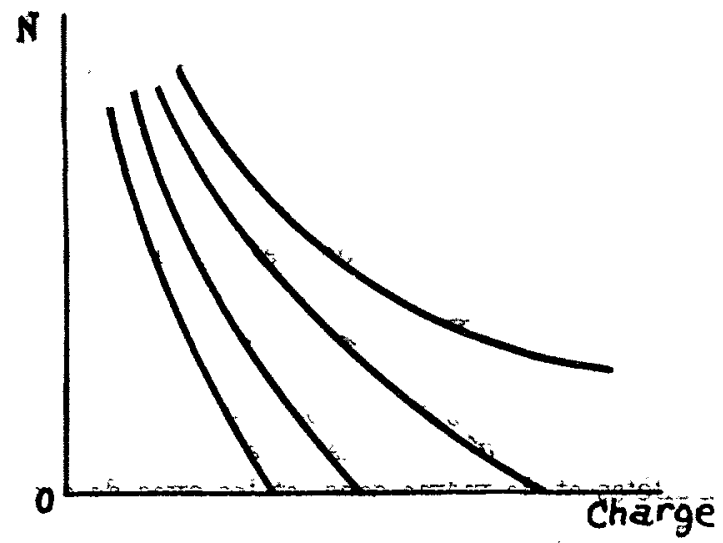

FIG. 22. - Moteur série à cc. (réglage par résistances).

Le moteur shunt avec réglage par le champ ou par résistance en série aura un faisceau de courbes d'allure (fig. 23 ou fig. 24.)

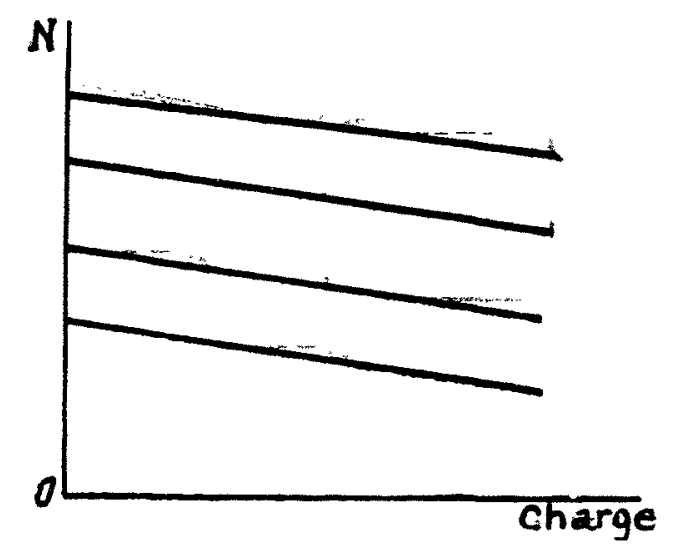

Fiód. 23. - Moteur shiunt cc. (rêglage par le champ)! Caractéristique shunt réglable.

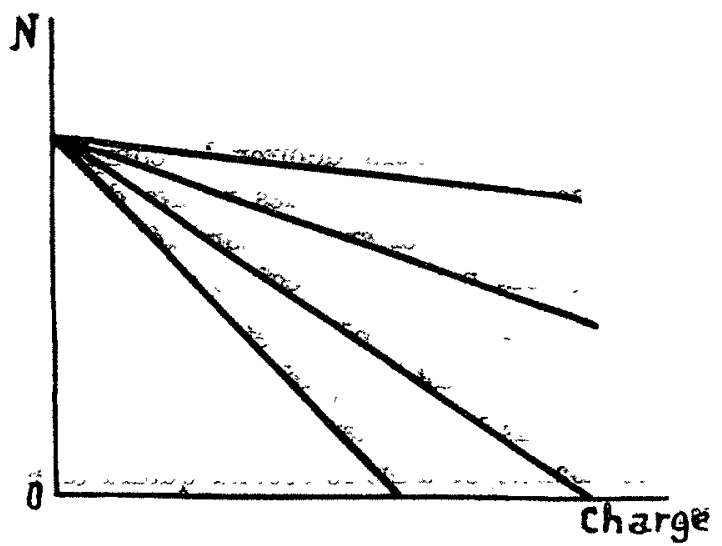

Fig. 21: - Moteu situt à ec. (rêglage par resistandes).

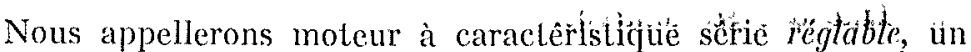

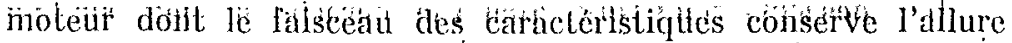

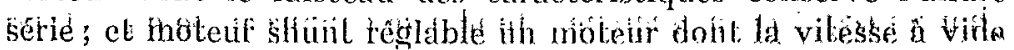
nist réglablè 
Enfin; üin miotétur cớnpónd réglablé sera un frioteur dont l'écart de vitesse entre vide et charge est régláble entré certaines limites $\mathrm{N}_{i}$ ét $\mathrm{N}_{2}$ (flg. 25).

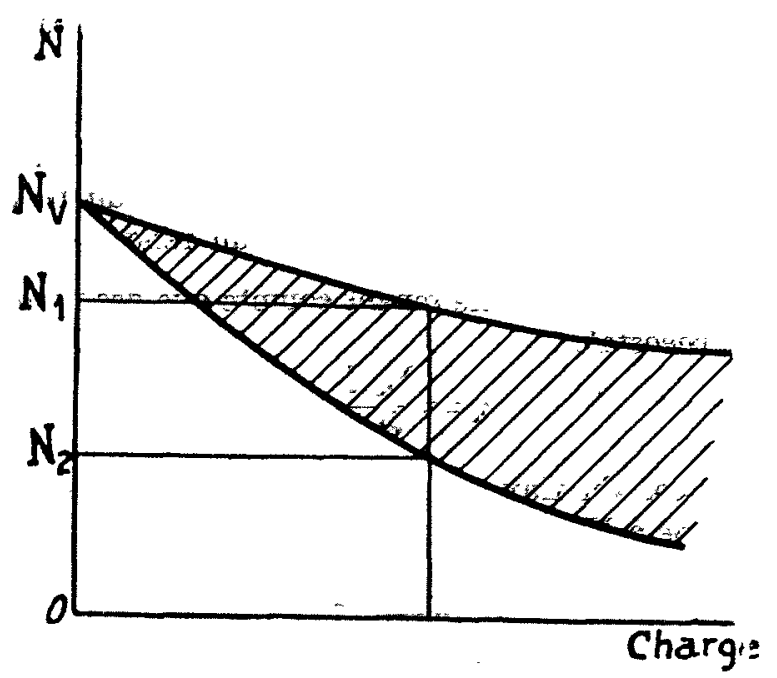

FIG. 25. - Caractéristique compound réglable.

La vitesse à vide du moteur compound peut être elle-même réglable (fig. 26).

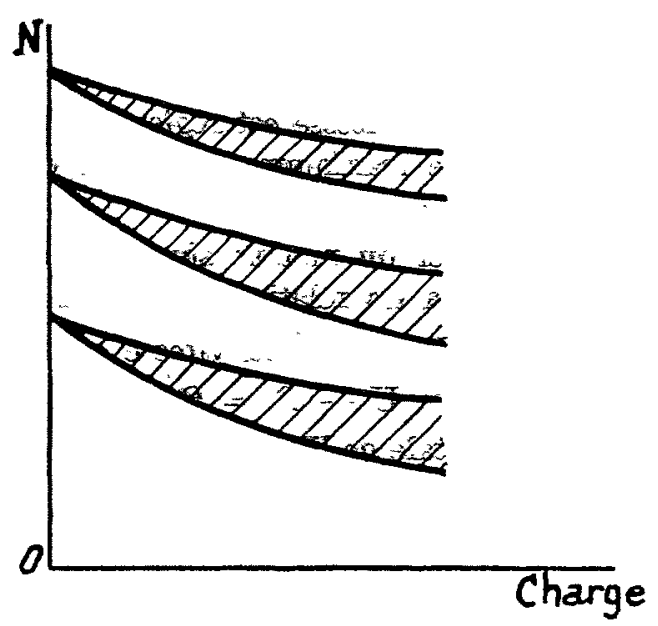

Fig. 26. - Caractéristique compound réglable à vitesse à vide réglable

Ainsi, le moteur défivatión à cöurant continu avec résistance en série et réglage par le champ est à vitesse à vidè et écart de vilesse réglables.

On voit donc que les différéntes allures de caractéristiques sont les suivantes :

1. - a) série simple (fig. 6);

b) sêrie réglable (fig. 21):

2. - a) shunt simple (fig. 5) ;

b) shünt réglable (fig. 23):

3. - - a) compound simple (fig. 7);

b) compound à chute de vitesse réglable (fig. 25) t

c) compound à vitesse à víde êt chute de vituessè rểglables (fig. 26).

Un exploitant qui désíte un moteur à vitesse variable doit, avant tout, indiquer laquelle de ces allites de caractéristique convient à lapplication qu' il th en vuei

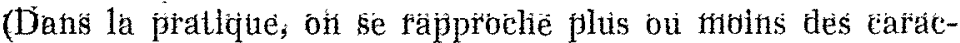
teristiques thériqués que notus vènons de définit. Ainsi; un moteur à caractéristique shunt réglable pourra avoir uite chute de vitesse en charge variable selon la vitesse â vide; ele...)

2. - Condittons d'emplöi tu motebr. - Conséquences au

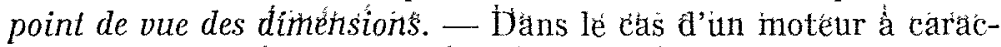
téristiçute réglable d'allưre donninée, le nibteur jeut êtré dimensioniné différenthinetit, seloón cé qu'on dn altend.

On peut, pâr exemple, désirer obténir a toutes les vitesśes

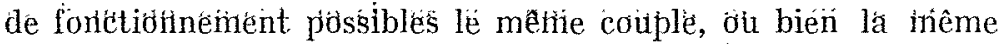
puissarice : oń dirá alors quiè le nonoteur eśt utilisé à vitèsse réglable ét coựle constánt, vitešse rẻglable ét puissatice constante.

Toute autré lói du cơple ên fonction dé là vltèssè peùt d'ail-

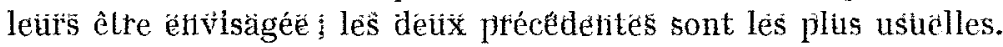

Il esst important de remarquer, que cette loi d'ulilisation

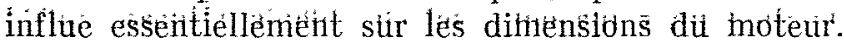

Nous raisonnerons en négligeant les phénomènes du second ordre (influence de la réaction d'induit et de la commutation sur les caractéristiques).

Un moteur est dimensionné pour son courant maximum (círcút électriquie) et son flux maximum (circuit magnétique).

En effet, les raisons de saturation et de pertes fixent les densités de flux limites, les raisons de rendement et de pertes fixent les densités de courant limites.

Pour un réseau de tension $U$, la puissance qui est $U$ I varie comme I : la puissance maxima fixe le courant maximum.

Dans le cas du réglage par le chantṕ, le couple est proportionnel à $\Phi \mathrm{I}$.

Comme $\mathrm{N}=\frac{\mathrm{U}-\mathrm{RI}}{a \Phi}$ (á étant une constante), en négligeant $\mathrm{R} I$ devant $U$ (ce qui rếvènt à supposer la force électromotrice constante), on voit que la vitesse varie en raison inverse du flux.

Le circuit magnétique doit être dimensionné pour le flux de basse vitesse. Il est mal utilisể en grande vitésse : le flux est. inférieur à celui que le circuit magnétique peút admettre.

Si le hióteử ést utilise à vitessè variăble èt cơujle constant, son circuit électriquè ñest, en outre, pas bien utilisé, car à la basse vitesse, le courant est inférieur à celui que le cuivre peut admettre : le cuivre n'est bien utilisé à toutes lés vitésses que dans le fonctionnement à vitesse variable et puissance confstante.

Ainsi, par exemple, soit à réaliser un moteur capablè â tbittés Ies vitesses entre 500 et 1.500 t: de la même puissance $100 \mathrm{CV}$.

Le moteur sera à 1.500 t. à son courant normal et au $1 / 3$ dủ flux normal. II sera à 500 tours à son courant normal et àu flủx rờrmảal:

Il aura les dimensions d'un moteur de $300 \mathrm{CV}$. à 1.500 t: èt sera donc trois fois plus gros qu'un moteur capablè seulzhent de $100 \mathrm{CV}$. à $1: 500 \mathrm{t}$.

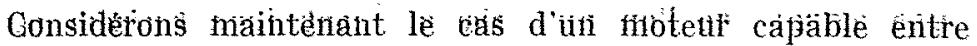
500 et 1.500 t: du coujple corfesporidaint â $100 \mathrm{CV}$ : à $1.500 \mathrm{t}$ :

Le ithotêti sêra à 500 toưs a son flux normal; et al $1 / 3 \mathrm{du}$ courant normal; il aura les mêmes dimensions qultẻ dáñ le cás précédéñt.

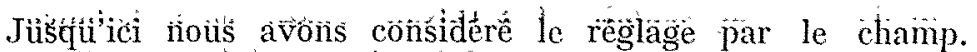

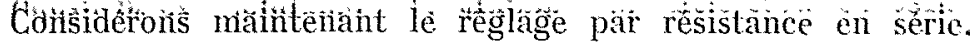

Soit $\dot{U}_{a}$ la tension aux bornes de l'induit. Supposons I donné,

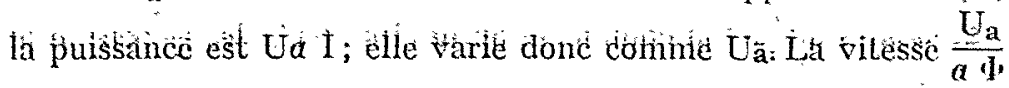
varie comme Ua, car $\Phi$ ést constant (cela est clair dans le cas 
du moteur shunt; et également avec moteur série car $\Phi$ ne dépend que de I qui est donné). Le couple, proportionnel à $\Phi$ I est donc constant.

Le moteur, utilisé avec résistances telles que le courant soit maintenu constant dans une certaine gamme de vitesses, est capable du même couple à toutes ces vitesses.

Pour un couple résistant donné, constant à toute vitesse, la vitesse se règle en modifiant la tension $\mathrm{U}_{\mathrm{a}}$ (la constance du couple impliquant automatiquement celle du courant).

Si le couple varie en raison inverse de la vitesse, on en déduit aisément la loi de variation du courant en fonction de la vitesse, et, par suite, celle de $\mathrm{U}_{\mathrm{a}}$ en fonction de la vitesse. Les valeurs maxima de $U_{a}$ et de $I$ déterminent les dimensions.

En conclusion, lorsqu'on commande un moteur à vitesse réglable, il faut indiquer non seulement la caractéristique désirée, mais la loi du couple normal désiré à chaque vitesse, puisque les dimensions de la machine en dépendent. Cette loi définit une courbe d'utilisation dont l'intersection avec la famille des caractéristiques couple-vitesse du moteur définit le point de fonctionnement de pleine charge à chaque vitesse.

La courbe d'utilisation du moteur sera : une droite (couple constant); une hyperbole (puissance constante) (fig. 27), cas d'un moteur shunt).

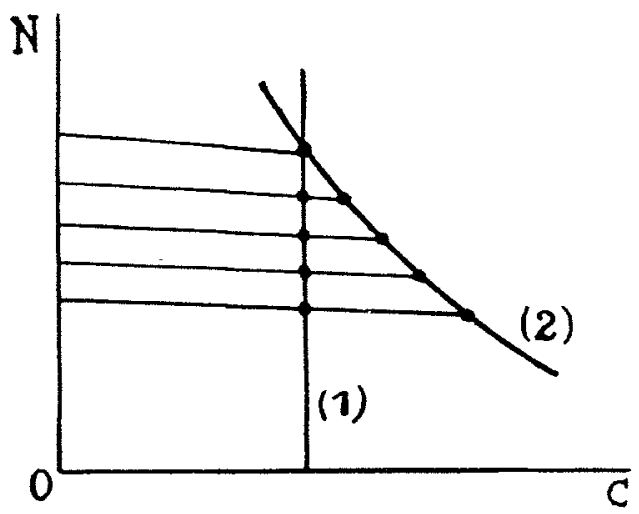

FIG. 27. - Moteur shunt utilisé à couple constant (1) et puissance constante (2).

3. - Limites au réglage possible de la vitesse. - Chaque genre de moteur possède ses limitations propres, pour des raisons qui peuvent être des raisons de commutation, de stabilité de fonctionnement, de rendement, etc.

Considérons le cas du réglage par le champ.

La tension de réactance pour une machine donnée est proportionnelle à $\frac{U \mathrm{I}}{\Phi}$, comme il résulte de la théorie de la commutation.

Par suite, elle augmente avec la vitesse pour un courant donné dans l'induit, $\Phi$ variant en raison inverse de $N$. Les vitesses supérieures que l'on peut réaliser, sont donc limitées par la nécessité de tenir la tension de réactance au-dessous d'une certaine valeur critique, ce qui est le critérium usuel de bonne commutation.

D'autre part, une machine très désexcitée manifeste un phénomène de distorsion. Nous dirons seulement, à ce sujet, que l'effet de distorsion est d'autant plus sensible, que le rapport des ampère-tours de réaction d'induit aux ampère-tours d'excitation est plus élevé ; il existe une valeur critique de ce rapport au-dessus duquel la commutation devient difficile, et pour lequel la machine devient sujette au flash par suite de l'augmentation de la tension locale entre lames.
En outre, la distorsion peul avoir pour conséquence une instabilité de vilesse.

D'autre part, l'excitalion maxima est limitée par la saturation; on est donc conduit, pour un courant donné, à maintenir le flux entre deux limites données; la pratique montre que le réglage de la vitesse de $1-3$ par le champ est une limite pratique, à puissance constante.

Considérons maintenant le réglage par résistance en série avec l'induit. Nous allons voir que l'étendue du réglage de la vitesse est limité ici par la question du rendement.

Le rendement est, en ne tenant compte que des perteṣ perdues dans le rhéostat,

$$
r_{1}=\frac{U_{a} I}{U I}=\frac{U_{a}}{U} \text {. }
$$

Dans le cas du flux constant, la vitesse est proportionnelle à $U_{a}$ et égale à $N_{\max }$ pour $U_{a}=U$, on aura :

Posons

$$
\eta=\frac{N}{N_{\max }} \text {. }
$$

$$
z=\frac{N_{\max }-N}{N_{\max }}
$$

Les pertes dans le rhéostat seront :

avec

$$
\mathrm{U} \mathrm{I}-\mathrm{U}_{\mathrm{a}} \mathrm{I}=\mathrm{g} \mathrm{P}_{\mathrm{a}}
$$

$$
\mathrm{P}_{\mathrm{a}}=\mathrm{UI} \text {. }
$$

On voit donc, que si la vitesse est réglée à une valeur faible par rapport à la vitesse maximum, les pertes deviennent très importantes, à couple constant.

Dans le cas particulier du moteur shunt, fonctionnant avec résistance en série, on a les relations suivantes :

dans la marche à vide $E=U$ et la vitesse est $N_{v}$, dans la marche en moteur $\mathrm{U}-\mathrm{E}=\mathrm{R} \mathrm{I}$, $\mathrm{R}$ étant la résistance totale de l'induit et du rhéostat.

$$
\frac{\mathrm{R} \mathrm{I}}{\mathrm{U}}=\frac{\mathrm{R} \mathrm{I}^{2}}{\mathrm{UI}}=\frac{\mathrm{J}}{\mathrm{P}_{\mathrm{a}}}=g
$$

$\mathrm{J}$ désignant les pertes Joule dans la résistance $\mathrm{R}$.

On a

$$
\begin{gathered}
a \mathrm{~N}_{\mathrm{v}} \Phi=\mathrm{U} \\
a \mathrm{~N} \Phi=\mathrm{U}-\mathrm{R} \mathrm{I}
\end{gathered}
$$

par suite

$$
\begin{gathered}
g=\frac{\mathrm{N}_{\mathrm{v}}-\mathrm{N}}{\mathrm{N}_{\mathrm{v}}}=\frac{\mathrm{J}}{\mathrm{P}_{\mathrm{a}}} \\
r_{\mathrm{i}}=\frac{\mathrm{N}}{\mathrm{N}_{\mathrm{v}}}
\end{gathered}
$$

la puissance mécanique sur l'arbre est, en négligeant les pertes propres du moteur

$$
\mathrm{P}_{\mathrm{m}}=\mathrm{U}_{\mathrm{a}} \mathrm{I}=\mathrm{P}_{\mathrm{a}}(1-g)=\mathrm{P}_{\mathrm{a}} \frac{\mathrm{N}}{\mathrm{N}_{\mathrm{v}}}=\mathrm{C} \frac{2 \pi \mathrm{N}}{60} .
$$

Dans le fonctionnement à couple constant, $\mathrm{P}_{\mathbf{a}}$ est constant quand la vitesse varie; mais les pertes augmentent rapidement quand le glissement augmente.

Ainsi, si la vitesse est réduite à $\frac{N_{\mathrm{v}}}{3}$, on aura :

$$
\mathrm{P}_{\mathrm{m}}=\frac{1}{3} \mathrm{~Pa} \quad \text { Joule rotor }=\frac{2}{3} \mathrm{~Pa}_{\mathrm{a}}
$$


En ne tenant compte que des pertes Joule rotor, le rendement $r_{i}$, est :

$$
\eta=0,33 \text {. }
$$

En tenant compte de toutes les pertes, le rendement réel est nécessairement inférieur à celte valeur.

Si le couple résistant normal que l'on doit vaincre à chaque vitesse est une fonction croissante de la vitesse $f(\mathrm{~N})$, alors, (1) désignant la vitesse angulaire de rotation, on aura :

$$
\begin{gathered}
\mathrm{P}_{\mathrm{m}}=\omega^{\prime} f(\mathrm{~N}) \\
\text { Joule rotor }=\frac{g}{1-g} \omega^{\prime} f(\mathrm{~N})
\end{gathered}
$$

Quand $\mathrm{N}$ diminue à partir de la grande vitesse, $g$ augmente, mais $f(\mathrm{~N})$ diminue. Le produit $g f(\mathrm{~N})$ n'augmente donc pas proportionnellement à $g$.

Le rendement aux basses vitesses est faible, mais les pertes étant alors une petite fraction de la puissance normale du moteur $\mathrm{P}_{\mathbf{n}}$ pourront être acceptables.

Ainsi, si le couple augmente comme $\mathrm{N}^{2}$, on aura :

$$
\begin{gathered}
P_{a}=P_{n}\left(\frac{N}{N_{v}}\right)^{2} \\
\text { Joule rotor }=P_{n}\left(\frac{N_{v}-N}{N_{v}}\right)\left(\frac{N}{N_{v}}\right)^{2}
\end{gathered}
$$

Pour $N=\frac{N_{v}}{3}$, on aura :

$$
\begin{gathered}
\mathrm{P}_{\mathbf{a}}=\frac{\mathrm{P}_{\mathrm{n}}}{9} \\
\text { Joule rotor }=7,4 \% \text { de } \mathrm{P}_{\mathrm{n}} .
\end{gathered}
$$

4. - Fonctionnement en couple négatif sur le réseau. - Dans le fonctionnement sur un réseau à tension constante, il importe d'examiner la manière dont les caractéristiques vitesse-couple se prolongent pour les couples négatifs, sans que l'on touche en rien au moteur, car, dans certaines applications, on peut

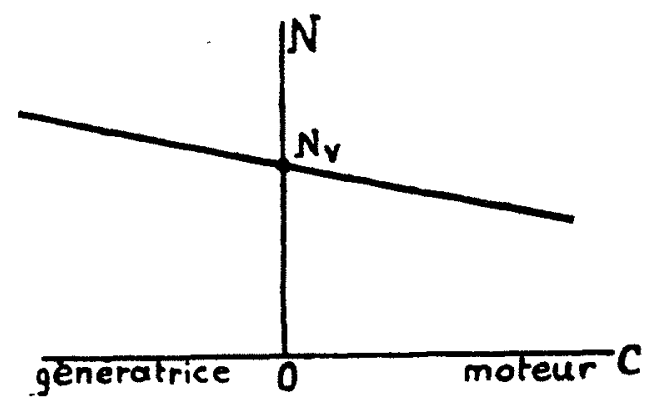

Fig. 28. - Moteur shunt cc. Couple en moteur et en génératrice.

ètre conduit à utiliser cette partie de la caractéristique, la machine restant connectée au réseau (fonctionnement en freinage continu).

Dans le cas du moteur shunt, pour un réglage donné de l'excitation, la vitesse à vide prend la valeur $\mathrm{N}_{\mathrm{v}}$. Si le couple devient négatif, la vitesse prend une valeur supérieure à $\mathrm{Nv}$.

On aura :

$$
\mathrm{E}-\mathrm{U}=\mathrm{R} \mathrm{I}
$$

$$
\frac{\mathrm{R} \mathrm{I}}{\mathrm{U}}=\frac{\mathrm{N}-\mathrm{N}_{\mathrm{v}}}{\mathrm{N}_{\mathrm{v}}}=-g
$$

$$
\text { et } \quad \frac{\text { Joule rotor }}{\mathrm{P}_{\mathbf{a}}}=g \text { en valeur absolue. }
$$

La puissance $\mathrm{P}_{\mathrm{a}}$ est fournie au réseau. La caractéristique en génératrice est le prolongement de celle que l'on obtient en moteur (fig. 28).

Il en est de même pour les différentes excitations.

Si la machine est entraînée par un moteur à couple normal constant et à vitesse variable, elle débite sur le réseau une puissance constante.

Les pertes Joule augmentent avec la vitesse, de la même quantité dont augmente la puissance mécanique avec la vitesse. Le courant débité par la machine ne dépend uniquement que du couple développé sur son arbre.

On peut utiliser la machine à vitesse variable, avec une résistance rotorique convenable. La vitesse minimum est la vitesse $\mathrm{N}_{\mathrm{v}}$, et l'on peut obtenir, pour un couple donné, des valeurs supérieures de la vitesse (fig. 29).

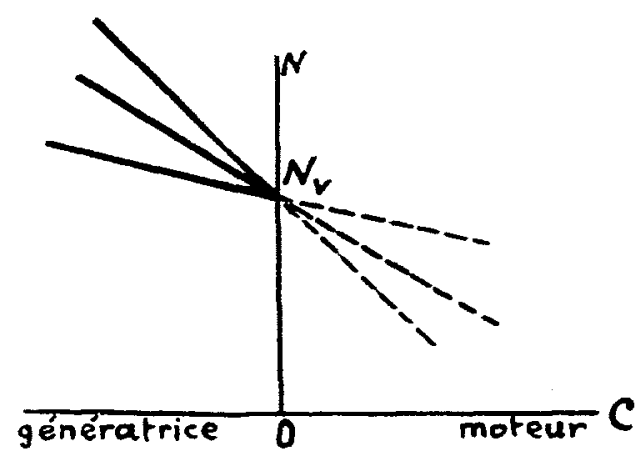

FIG. 29. - Moteur shunt cc. - Réglage par résistance en série dans la marche en génératrice.

Pour une très grande résistance et un couple donné, la machine s'emballe.

La difficulté de commutation augmentant avec la vitesse peut interdire ce mode de fonctionnement en génératrice au delà d'une certaine vitesse.

Quand on passe de la pleine charge en moteur à la pleine charge en génératrice, le glissement est égal au double de la chute ohmique exprimée en pour cent de la tension à vide.

On peut encore réaliser d'une autre manière le fonctionnement en couple négatif du moteur shunt : on croise les connexions * entre les inducteurs et l'induit. Le courant pris au réseau prend la valeur $\frac{U+E^{\prime}}{R}$ et le moteur fonctionne en couple négatif en absorbant de la puissance sur le réseau. Comme le courant est considérable, il importe de le limiter par une résistance appropriée dont la valeur est plus élevée que la résistance de démarrage.

Le fonctionnement en couple négatif du moteur série sur le réseau présente une difficulté particulière :

Ce fonctionnement, qui n'est possible qu'après avoir croisé les connexions entre l'inducteur et l'induit, est un fonctionnement instable.

En effet, si pour une raison accidentelle quelconque, la tension $\mathrm{U}$ du réseau vient à diminuer, le courant I augmente. En passant dans l'excitation série, il détermine une augmentation de $\mathrm{E}$ qui a pour conséquence un nouvel accroissement de $I$. 
Inversement, si $\mathrm{U}$ diminue, $\mathrm{E}$ dithititic; s'dnnule, le courant $I$ se rétablit en sens inverse; E s'invetse et s'ajoute à la tension de ligne, le courant I alleinl une valeur très élevéc.

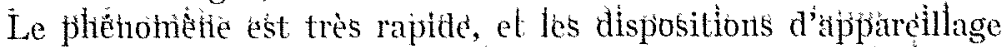

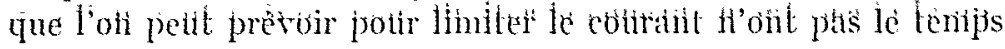
d'agir.

5. - Fonctionnement en couple négatif indépendamment du irsedu. - Leès nidehines à courant continu pecivent fonctiotiner

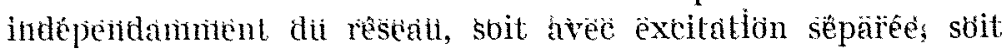
en utilisanl la propriété de l'auto-excitation. Eü êfrèt; ón säit que, sous réserve de certaines conditions de résistances; la machine est susceptible de s'exciter spöntanément. Avec une machine à flux constant (machine shunt ou machine série sat urée), je couple négatif ainsi obtenu est proportionnel à la vilesse et en raison inverse de la résistance aux bornes de l'induil. On né peùt dờc pas freiner juscfủă l'artêt.

6. - Réalisation d'une gamme étendue de vitesses. - Pour augmenter l'étendue du réglage de la vitesse; il faut renoncer à l'alimentalion du moteur par une tension conslante; on est alors conduit à intercaler entre le réseau et le moleur des appareils auxiliaires, et à remplacer le moteur sith́ple par un groupe de machines plus ou moins complexe.

En courant continu, un transformatetir de tension permettant de transformer la tension conistanite dì réseau en une tension variable entre des limites donnéés, peut être réalisé de différentes manières, à l'aide de machínes tournantes :

a) Transformateur Léotiard (fig. 30). - On alimente par le

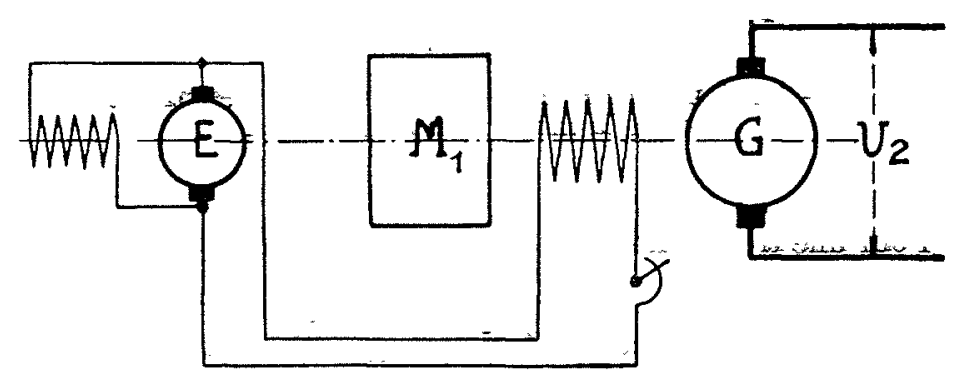

Fig. 30. - Transformateur_Léonard.

G Génératrice à excitation réglable.

$\mathrm{M}_{1}$ Moteur d'entraînement de la générátrice, à vitesŝ̉e sensiblemient constantê.

E Extitatrice de la génératríce.

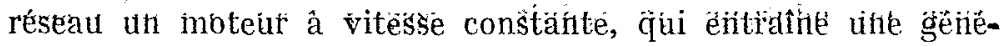
ratrice à tension réglable par l'excitation.

Dans ce cas, le réseau peut être à courant continu ou alternâtif èt la tension d'alimentation du moleur principal peut êtire réglée de $-\mathrm{U}_{2}$ à $+\dot{\mathrm{U}}_{2}$.

b) Survolteur-dévolteur (fig. 31). - On utilise un sutrvótieúr de tension $u$ en série avec le réseau de tension $\mathrm{U}$; ce qui perinet de disposer, par réglage de l'excilation du survolteur; d'une tension $\mathrm{U}_{2}$ variable de $\mathrm{U}-u$ à $\mathrm{U}+u$.

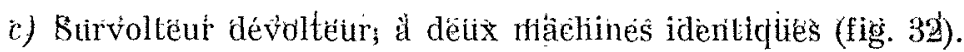
- On utilise deux machines à excilation séparể prise sùr le réseau, dont les induits sont reliés en sćrie sur le réseau $U$ et accouplés stur un même arbre.

Les excitations sont conjuguées te telle façón que les flux soienl bujburs complémentaires dans les deux induits la somma dès f: e. m. est lute conslatite, et les teinsions ruspectives des induits sont complémentidires:

Cette machine double tourne à vitesse constante par ses propres moyens.

On connecte le récepteur aux bornes de l'un des induils;

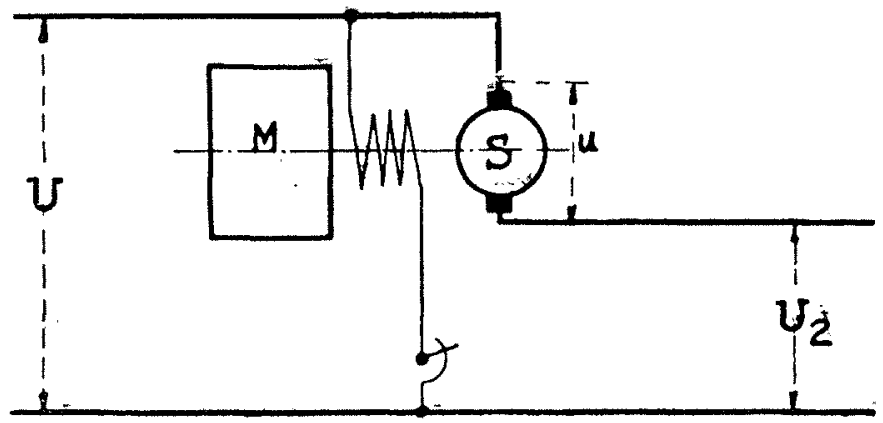

Fra. 31. - Survolteur devoltedir.

$S$ Survolteur à excitation reglable de tension u.

M Moteur d'entrainènent du survolteur; a vitesse sensiblément. constante.

en variant l'excitation conjuguée, on peut faire passer la tension $\mathrm{U}_{2}$ de la valeur zéro à la valeur $\mathrm{U}$.

Les systènès (b) è (c) constituent eil sómme des aulotransformateurs tournant à courant continu; le système (a) est. un véritable transformateur.

Avec le réglage Léonard, on peut régler facilement la vitesse d'un moteur à excitation séparée: Ce moteur est bien utilisé dans le fonctionnement à couple constant : c'est-à-dire que si l'on veut un moteur capable à toutes les vitesses entre 500

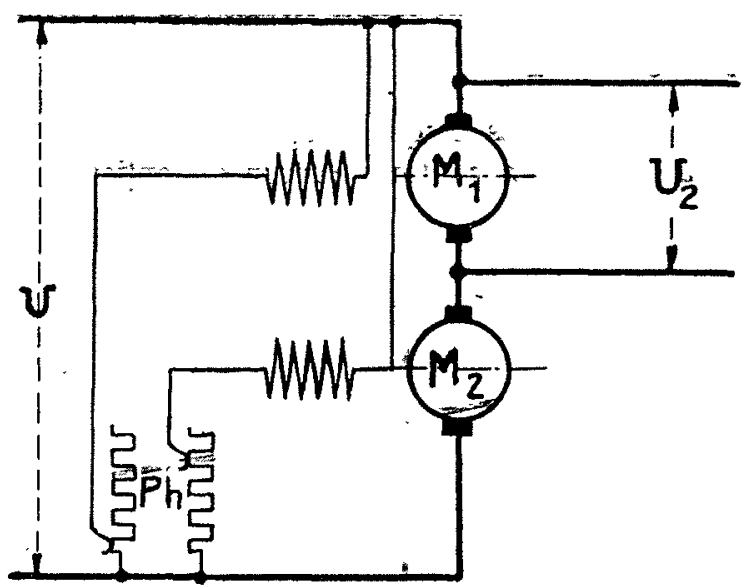

Fig: 32. - Attotransformateur à courant continu.

$\mathrm{M}_{1}, \mathrm{M}_{2}$ Induits accouplés sur un même arbre.

Rh Rhéostats conjúgués.

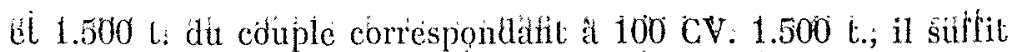
de prendre tu motetir de $100 \mathrm{CV}$. à $1.500 \mathrm{t}$. Il fonclionnerà à tenșion variable; flux el courants constants : par suite, il sera trois lois plus petit que si le même réglage devait être oblenu par l'excilation du moteur.

7. - Conditions du démarrage. - - Avec les moleurs série el shunt, le couple qu'il est possible d'obtenir au démarrage est extrêmement élevé. En effet, avéc ces hoteurs, le couple ne dépend que du coutant, et lo coturant do demarrage est le rapporl 
de la tension du réseau a la résistance interne du moteur. Le rapporl du courant de démarrage au courant normal peul atteindre 20 ou 30 ; il est d'aulant plus élevé que le moteur est plus importanl. Le rapport du couple de démarrage au couple nomal est egal au rapport des courants pour le moteur shunt, ou le molcur séric saluré.

Il esl nécessaire d'intercaler une résistance en série au démarrage, pour éviter la surintensilé et la brutalité de l'à-coup de couple.

Pour les machines à courant conlinu (sauf celles à grande rúsistance propre de l'induil), le couple que l'on peul oblenir au démarrage est donc loujours plus grand que celui que peut demander la machine à entraîner.

Dans le cas où la condition de démarrages fréquents est imposée à un moteur, le moteur séric ou compound concordant doit che préféré au moleur shunt, car le couple augmente alors plus rapidement que le courant, lant que la saluration n'est pas alleinte. Par exemple, si $\mathrm{Cd}=2 \mathrm{C} n$ on prendra au démarrage

2 fois le courant normal avec moleur shunt.

1,4 fois le courant normal avec moteur série non saturé.

8. - Variation de la tension du réseau. - - Nous supposerons unc variation relativement lente, car nous excluons de la présente élude les phénomènes de régime transitoire.

Le courant absorbé par le moteur est délerminé par la relation

$$
\mathrm{I}=\frac{\mathrm{U}-\mathrm{E}}{\mathrm{R}}
$$

par suilc, si U varie d'une quantité donnée, la variation de I dépend de celle de $\mathrm{E}$.

Une relation supplémentaire est nécessaire pour déterminer le problème.

Supposons que le moleur fonclionne sur couple résistant constant, indépendant de la vitesse.

Dans le cas du moteur série ou à excitalion séparée, le couple ne depend que du courant : donc le courant reste constant. La vitesse est liée par une relation à la lension $U$, elle varie proportionnellement à $U$ si la chute ohmique est négligeable. Dans le cas d'un moteur shunt, si $U$ baisse, l'excitation baisse, avec moleur non saturé. L'excitation diminuant, I doit augmenter d'aulant pour conserver le couple. La vilesse varie à peine. Au contraire, si le moteur est saturé, I varie peu et la vilesse baisse.

D'une laçon générale, avec le moteur shunt, l'influence d'une variation de tension sur la vitesse que prend le moleur pour un courant donné, dépend de la saluration, de la chute ohmique el de la réaction de l'induit. Selon les cas, la vitesse augmente ou diminue.

Dans le cas du fonctionnement du moteur à excitation séparée en couple négalif sur le réseau avec connexions normales en moteur, le courant débité varie quand la tension varie, une varialion de tension importante domnera donc des variations de courant tout à fait inacceptables; ce mode de freinage est rejeté en traction pour cetle seule raison.

9. - Sensibilité de la caractéristique. - Si l'on veul réaliser une caracléristique de couple moteur en fonction de la vitesse qui, pour un réglage domné, soit exactement déterminée, il faut considérer, outre les variations accidentelles de tension du réseau, la variation de l'échauffement de la machine ou des rhéoslats avec lesquels elle fonctionne.

Dans cerlains cas, ces variations modifient notablement la caractéristique. Ainsi, avec le moteur shunt, quand les électros s'échauffent, l'excitation diminue el, par suile, la vitesse augmente. Si le moteur entrâne un ventilateur, le moleur réglé initialement pour sa charge normale se trouvera bientôt surchargé.

Avec un moteur à réglage par résistance dans l'induil, surmontant un couple donné, la vitesse au voisinage de la pleine charge diminue relativement beaucoup quand l'échauffement de la résislance augmente (si celle-ci n'est pas constituée par un métal à très faible coefficient de tempéralure).

10. - Possibilités de réalisation. - Les machines à courant continu peuvent être réalisées pour toutes les tensions courantes jusqu'à $600 \mathrm{~V}$.; il est possible d'atteindre 1.500 et même $3.000 \mathrm{~V}$., mais les phénomènes transitoires de court-circuit et de surtensions premnent alors une importance qui rend le fonctionnemént plus délicat.

Les puissances de quelques centaines ou milliers de chevaux peuvent être atteintes, el les vitesses depuis quelques tours jusqu'à quelques milliers de tous par minute. La polarité de la machine est a priori arbilraire, elle est déterminée :

$1^{\circ}$ Par la fréquence dans l'induit (qui ne doit pas dépasser une certaine limite, pour la bonne utilisation de la machine).

$2^{\circ}$ Par la puissance par pôle; pour laquelle ìl esl difficile dé dépasser la valeur de 2 à $300 \mathrm{CV}$. environ (pour raisons de commutation);

$3^{\circ}$ Par des considérations diverses d'économie, de section de cuivre (pertes supplémentaires), de poids (le poids de l'armature de l'inducteur diminuanl rapidement quand la polarité augmente), etc.

En outre, les difficultés de construction inhérentes à la présence du collecteur rendent difficile la construction de machines de puissánce élevée à très faible tension pour de grandes vitesses angulaires.

11. - Possibilités des moteurs à courant continu. - Récapitulation. - Finalement, les moteurs à courant continu permettent d'obtenir toutes les allures de la caractéristique couple-vitesse.

10 Le moteur shunt à réglage par le champ est utilisé au mieux à puissance constante dans toute la gamme des vitesses. L'ulilisation de la machine est faible si la gamme des vitesses est étendue, surtout si on l'emploie à couple constant, c'est-à-dire que la machine est de grandes dimensions comparée à celle qui ferait le mème service étant alimentée par un groupe Léonard.

La gamme des vitesses réalisable est environ 1 à 3 , à couple ou à puissance constante.

Dans la pratique, le glissement pour un couple domné diminue d'abord quand la vitesse baisse, passe par un minimum, puis augmente à nouveau.

Le moteur shunt à réglage par résistance en série avec l'induit, est utilisé au mieux à couple constant. Le glissement au couple normal est minimum sans résistance ( 2 à $5 \%$ ) et croît jusqu'à l'unité quand la résistance augimente.

Les pertes Joule sont $g \mathrm{P}_{\mathbf{a}}, \mathrm{P}_{\mathbf{a}}$ étant constant. Le rapport $\frac{\mathrm{N}}{\mathrm{N}_{\mathrm{v}}}$ est une limite supérieure du rendement. 
Toutes les vitesses peuvent ètre obtenues.

On peut combiner les deux reglages, et fonclionner à puissance constante de 1 à 3 par le champ, et à couple constant de 0 à 1 par résistances. On aura ainsi le faisceau de caractéristiques de la fig. 33.

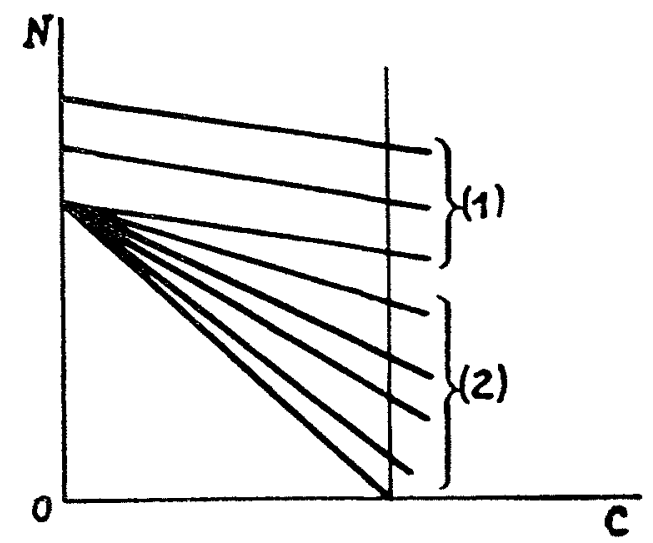

FIG. 33. - Moteur shunt à couple constant réglage par le champ (1) et par résistances (2).

Toutes les caractéristiques, sensiblement rectilignes, se prolongent vers les couples négatifs.

Il est des cas où l'on peut hésiter entre les procédés de régulation de la vitesse. Considérons, par exemple, le cas d'un moteur shunt pour ventilateur, la vitesse devant être réglée de 1 à 3 .

On peut employer le réglage par le champ, ou le réglage par résistance en série, la caractéristique convient aussi bien. Il convient de comparer les dimensions du moteur et l'énergie prise au réseau aux différentes vitesses dans les deux cas.

La solution la plus économique n'est pas immédiatement évidente.

$2^{\circ}$ Le moteur série à réglage par le champ (shuntage) est utilisé au mieux à vitesse variable et puissance constante, avec une gamme de vitesses de 1 à 3 dans le fonctionnement à couple ou puissance constante.

Le moteur série à réglage par résistance est utilisé au mieux à couple constant; la perte Joule est $g \mathrm{~Pa}, \mathrm{~Pa}_{\mathrm{a}}$ étant constant. Toutes les vitesses peuvent être obtenues.

$3^{\circ}$ Avec un moteur shunt plus ou moins compoundé, on peut obtenir des caractéristiques d'allure intermédiaire. En modifiant le nombre des spires utilisées sur les deux enroulements d'excitation, on passera d'une forme à une autre.

On peut ainsi réaliser un moteur compound à chute de vitesse réglable, au prix d'artifices lels que le shuntage de l'excitation série assurant une variation de l'excilation sous l'action de la charge.

$4^{\circ}$ Le moteur shunt, alimenté par transformateur tournant, permet de réaliser une variation de vitesse de 1 à 10 à couple constant.

Mais il est difficile de dépasser cette limite, car on obtient alors aux faibles vitesses un glissement assez élevé. II se manifeste en outre certains phénomènes d'instabilité de la vitesse à période très lente, pour lesquels l'hystéris en particulier joue son rôle. En outre, la chute de tension aux balais devient une importante fraction de la chute ohmique totale, et le calcul doit en tenir compte.

Le tableau suivant résume ce qui précède, et précise les possibilités de la machine à courant continu au point de vue du réglage de la vitesse.

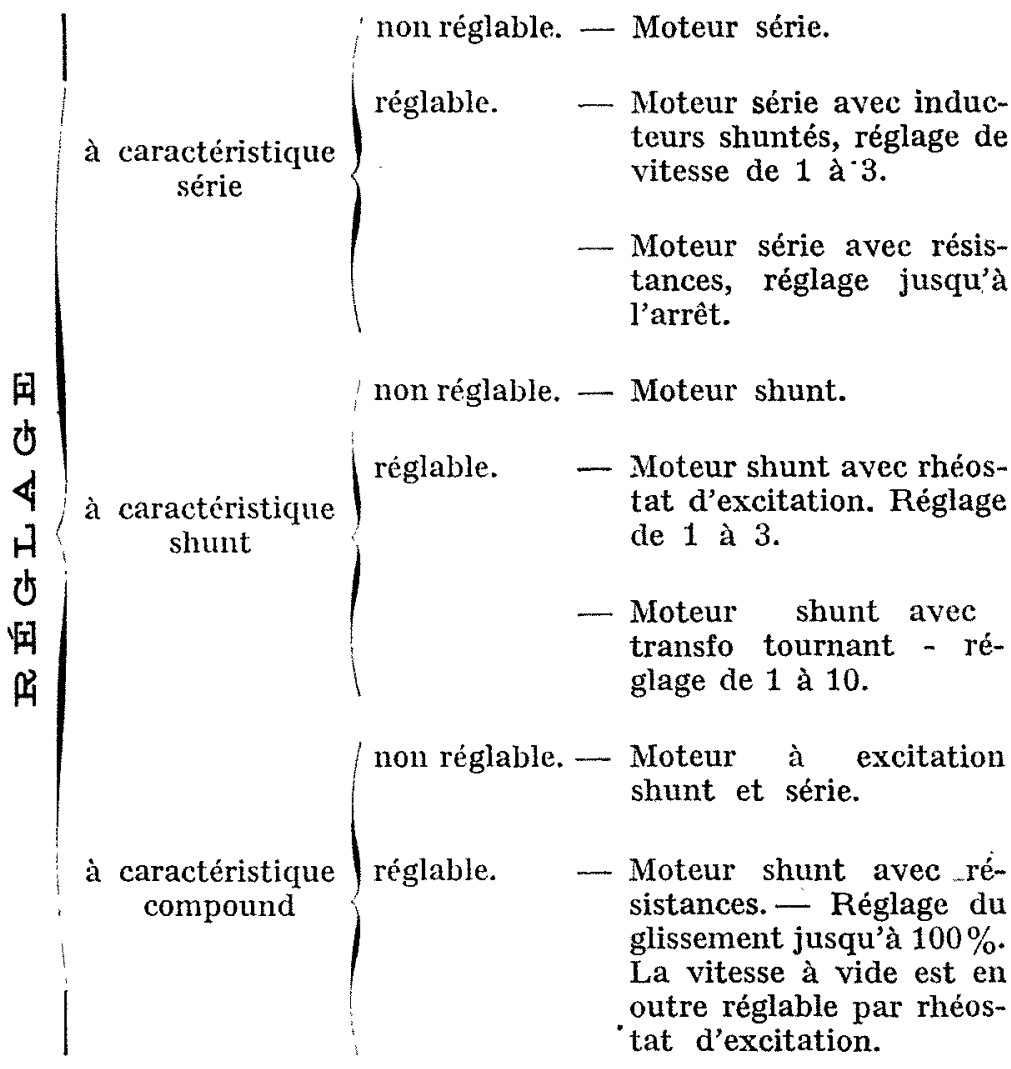

(A suivre.)

(Extrait de Jeumont, avril-juin 1928). 